\title{
A GENERAL MODEL OF INFORMATION SHARING IN OLIGOPOLY*
}

\author{
by \\ Michael A Raith \\ London School of Economics and Political Science
}

Contents:

Abstract

1. Introduction

2. General Model

3. Nash Equilibrium of the Oligopoly Game

4. The Incentives to Share Information

5. Concluding Remarks

A. Proofs of Lemmas and Propositions

B. Definitions of Symbols

References

Discussion Paper

No. TE/93/260

March 1993
The Suntory Centre

Suntory and Toyota International Centres for

Economics and Related Disciplines

London School of Economics and Political Science

Houghton Street

London WC2A $2 \mathrm{AE}$

Tel.: 020-7955 6698

* I am especially indebted to Frank Bickenbach and Georg Nöldeke for numerous valuable comments and suggestions on the previous draft. Furthermore, I would like to thank Patrick Bolton, Benny Moldovanu, Urs Schweizer, Avner Shaked, John Sutton, and seminar participants in Bonn, Louvain-la-Neuve, and Jerusalem for helpful discussions and comments. An earlier version of the paper benefited from comments by Rembert Birkfeld, Clemens Esser, Guido Friebel, and Mark Spoerer. All remaining errors are my own. Finally, financial support from the Sonderforschungsbereich 303 (University of Bonn) and the Deutscher Akademischer Austauschdienst is gratefully acknowledged. 


\begin{abstract}
Under which circumstances do oligopolists have an incentive to share private information about a stochastic demand or stochastic costs? We present a general model which includes virtually all models of the existing literature on information sharing as special cases.

The analysis reveals that in contrast to the apparent inconclusiveness of previous results some simple principles determining the incentives to share information can be obtained. Most existing results are generalised and some interpretations are corrected, leading to a single general theory of the topic.
\end{abstract}

Keywords: Oligopoly, information sharing, stochastic demand, stochastic costs.

(C) Michael Raith. All rights reserved. Short sections of text, not to exceed two paragraphs, may be quoted without explicit permission provided that full credit, including $\odot$ notice, is given to the source. 


\section{Introduction}

Theoretical research on information sharing in oligopoly was pioneered by Novshek and Sonnenschein (1982), Clarke (1983) and Vives (1984). Over the following decade, numerous contributions on this topic have appeared. While the models analyzed vary along several dimensions, their basic structure is the same, which is also the structure of the model to be analyzed in this paper:

We consider an oligopoly where firms face either a stochastic intercept of a linear demand function or a stochastic marginal cost. In the most general case, the firms produce heterogeneous goods, where the random demand or cost changes may be different for each firm, but usually are correlated. The deviation of the vector of demand intercepts/costs from its mean, henceforth called "State of Nature", is unknown to the firms.

Instead, each firm receives a private signal which contains information about the true State of Nature. This signal can be regarded as a sufficient statistic of a number of single observations - market research, reports of salesmen etc. For example, firms might receive noisy signals about the intercept of a common demand function, or they might know their own costs exactly, but not the costs of the rival firms.

Private information may be exchanged, e.g. by means of a trade association collecting and disseminating data acquired by the firms of an industry. We assume that firms commit themselves either to reveal their private information to other firms or to keep it private before receiving any private information, hence the decision on the revelation behavior cannot be made dependent on the realization of the signal. ${ }^{1}$

In the last stage, called the "oligopoly game", firms noncooperatively set prices or quantities according to their private and the revealed information so as to maximize expected profits.

Two approaches to analyze the revelation behavior of firms have been discussed in

1 Many authors have also considered partial information disclosure, which in essence means that only a subset of the observations underlying the private signal is revealed, in contrast to the true private signal as a sufficient statistic of all observations. 
the literature: In the simpler case, we determine under which circumstances industrywide contracts on information sharing are profitable by comparing the equilibria and expected profits in the cases of no information sharing and complete sharing. In the other variant, which has received most attention in the literature, firms simultaneously and independently decide on their revelation behavior, e.g. by entering into contracts with their trade association, which may lead to some firms revealing their information and others concealing it. The first approach involves the analysis of a one-stage oligopoly game under different assumptions on information sharing, while the other approach leads to a two-stage game, where firms first make their revelation decisions and then play the oligopoly game.

Several questions arise: How do prices/quantities and expected profits with and without information sharing depend on (i) the type of competition (Cournot or Bertrand), (ii) the characteristics of goods (substitutes or complements, homogeneous or heterogeneous products), (iii) the type of uncertainty (demand or cost uncertainty), (iv) the correlation of the stochastic demands/costs among the firms, (v) the precision of the signals, and (vi) the correlation of the signals? How do the expected profits for a given informational structure influence the incentives for firms to exchange private information in the first place?

Excluding collusion in the price/quantity setting stage, information sharing has two effects from the viewpoint of the firms: each firm is better informed about the prevailing market conditions, and the homogenization of information among firms leads to a change in the correlation of the strategies. While the first effect is presumably profitable, the second may be unprofitable, leaving the net effect of information sharing on the expected profits ambiguous.

I will not review in detail the various contributions in the literature addressing these questions. Instead, the particular assumptions and results on the incentives to share are displayed in Table 1 (see Vives [1990] for a brief survey of the literature).

It has been noted by Vives (1990) and can easily be checked in the table that 
Table 1: Previous work on the incentives to share information: assumptions and results (for substitute goods)

\begin{tabular}{|c|c|c|c|c|c|c|c|c|}
\hline \multicolumn{4}{|c|}{ cost or demand uncertainty? } & & & \multicolumn{3}{|c|}{ State of Nature (\# of components) } \\
\hline \multicolumn{4}{|c|}{ product differentiation? } & & & & \multicolumn{2}{|c|}{ private signal } \\
\hline Cournot or Bertrar & & & & & & & \multicolumn{2}{|c|}{ revelation of signals } \\
\hline \# of firms & & & & & & & & results \\
\hline Novshek/Sonnen- & & & & & & & & \\
\hline schein (1982) & 2 & $\mathrm{C}$ & & $\mathrm{d}$ & 1 & noisy & partial, asymm. & ambiguous \\
\hline Clarke (1983) & $\mathbf{n}$ & $\mathrm{C}$ & & d & 1 & noisy & all firms or none & no sharing \\
\hline Fried (1984) & 2 & $\mathrm{C}$ & & c & $\mathrm{n}$ & perfect & asymm. & sharing \\
\hline Vives (1984) (1) & 2 & $\mathrm{C}$ & $\mathrm{x}$ & $\mathrm{d}$ & 1 & noisy & partial, asymm. & no sharing \\
\hline Vives (1984) (2) & 2 & B & $\mathrm{x}$ & d & 1 & noisy & partial, asymm. & sharing \\
\hline Gal-Or (1985) & $2 / n$ & $\mathrm{C}$ & & $\mathrm{d}$ & 1 & noisy & partial, asymm. & no sharing \\
\hline Li (1985) (1) & $\mathrm{n}$ & $\mathrm{C}$ & & d & 1 & noisy & asymm. & no sharing \\
\hline $\mathrm{Li}(1985)(2)$ & $\mathrm{n}$ & $\mathrm{C}$ & & c & $\mathbf{n}$ & perfect & asymm. & sharing \\
\hline Gal-Or (1986) (1) & 2 & $\mathrm{C}$ & $\mathrm{x}$ & c & $\mathbf{n}$ & noisy & partial, asymm. & sharing \\
\hline Gal-Or (1986) (2) & 2 & B & $\mathrm{x}$ & c & $\mathbf{n}$ & noisy & partial, asymm. & no sharing \\
\hline Shapiro (1986) & $\mathbf{n}$ & $\mathrm{C}$ & & c & $\mathrm{n}$ & perfect & all firms or none & sharing \\
\hline Sakai (1986) (1) & 2 & $\mathrm{C}$ & $\mathrm{x}$ & d & $\mathrm{n}$ & perfect & asymm. & sharing \\
\hline Sakai (1986) (2) & 2 & B & $\mathrm{x}$ & d & $\mathrm{n}$ & perfect & asymm. & sharing \\
\hline Kirby (1988) & $n$ & $\mathrm{C}$ & & d & 1 & noisy & all firms or none & $\begin{array}{l}\text { depending on } \\
\text { parameters }\end{array}$ \\
\hline Sakai/ & & & & & & & & \\
\hline Yamato (1989) & $\mathrm{n}$ & $\mathrm{C}$ & $\mathrm{x}$ & d & $\mathrm{n}$ & perfect & all firms or none & sharing \\
\hline Hviid (1989) & 2 & $\mathrm{C}$ & & d & 1 & noisy & asymm. & $\begin{array}{l}\text { depending on } \\
\text { parameters }\end{array}$ \\
\hline
\end{tabular}


the results concerning the incentives to share information seem to depend sensitively on the specific assumptions of the model: A change from Cournot to Bertrand, from substitutes to complements, from demand to cost uncertainty, or from a "common value", referring to a one-dimensional State of Nature, to "private values", referring to an $n$-dimensional State of Nature for $n$ firms, may lead to completely different outcomes. More disturbingly, however, apparently similar models often lead to contrasting results. Two points shall illustrate that the existing literature cannot satisfactorily explain this diversity of results.

(i) Vives (1984), Gal-Or (1985), and Li (1985) show that in a Cournot oligopoly with homogeneous goods and demand uncertainty firms do not share information in the equilibrium of the two-stage game described above. In contrast, Fried (1984), Li (1985), and Shapiro (1986) show that in a Cournot market with uncertainty about private costs firms completely reveal information in the equilibrium. Several authors (Fried 1984, Li 1985, Gal-Or 1986, Vives 1990) have attributed this contrast to the difference between a "common value", e.g. the intercept of a common demand function, and "private values", e.g., different marginal costs for the firms. However, there is an inconsistency in this interpretation. The results of Fried, Li, and Shapiro for "private values" hold even if the correlation of marginal costs approaches unity, although economically, this situation is equivalent to a model with a common value. This is disturbing as in this class of models, we should expect profits to vary continuously with the underlying parameters. The resolution of this problem in Sections 2 and 4 will show that the private/common value distinction is indeed not the driving force.

(ii) Vives (1984) shows that in a duopoly with differentiated products and demand uncertainty, a change from substitutes to complements or from Cournot to Bertrand yields opposite results as to the incentives to share information. This may be attributed to a change in the slope of the reaction curves. However, in the private-values, cost-uncertainty model of Gal-Or (1986) there is only a difference between Cournot and Bertrand but not between substitutes and complements. Finally, in Sakai's (1986) model firms always share information, regardless of whether they set prices or quantities, 
or whether the goods are substitutes or complements. Hence from these results, very little can be concluded about the role of the type of competition and the characteristics of goods.

To summarize, there seem to be no general principles which underlie the existing results on information sharing in oligopoly. Even worse, only little seems to be known about the forces driving each particular result. It will be shown in this paper that these problems can all be resolved.

Thus the objectives of this paper are the following: (i) to show how a large number and variety of oligopoly models to be found in the literature can be analyzed at once within a single general model, leading to generalizations of most results, (ii) to argue that previous interpretations of information sharing models are not always consistent with the formal analyses, (iii) to formulate simple principles which determine the incentives to reveal information, and (iv) to provide new explanations for the general results.

The paper is organized as follows: Section 2 describes the general model. In Section 3 , the equilibrium strategies and expected profits for the oligopoly game are derived. In Section 4 we analyze the incentives to share information. Section 5 summarizes the results. The welfare effects of information sharing, discussed by some authors, have been analyzed in the model framework of this paper, but the results have not been included in this paper. In the last section, I will briefly turn to this issue.

\section{General Model}

In this section, a stochastic n-firm oligopoly model with private information is introduced at its most general level. In later sections, when we analyze particular aspects of information sharing, we will have to impose additional symmetry assumptions.

We first discuss the main elements of the model: the State of Nature, private information, information sharing, and strategies and payoffs. Subsequently, explicit game formulations are given. 
State of Nature: The State of Nature is denoted by the random variable $\tau=$ $\left(\tau_{1}, \ldots, \tau_{n}\right)^{\prime}$, where $\tau_{i}$ is the deviation of either the marginal cost or the intercept of a linear demand function of firm $i$ from its mean, depending on the type of uncertainty under consideration (the prime denotes transposition). ${ }^{2}$ Note that for demand uncertainty, the intercepts may be different for each firm as well as for cost uncertainty. The vector $\tau$ is normally distributed with $\mathrm{E}\left(\tau_{i}\right)=0, \operatorname{Var}\left(\tau_{i}\right)=t_{H}>0$ and $\operatorname{Cov}\left(\tau_{i} \tau_{j}\right)=t_{N} \in\left[0, t_{H}\right](j \neq i)$. Hence the covariance matrix of $\tau$ is $\mathrm{T}$, where $\mathrm{T}$ is $\mathrm{n}$-dimensional with $t_{H}$ on the main diagonal and $t_{N}$ everywhere else. Via the parameter $t_{N}$ the correlation of the $\tau_{i}$ can take any value between -1 and 1 , however, when interpreting later results we will assume that the $\tau_{i}$ are not negatively correlated, i.e. $t_{N} \geq 0$.

Private information: For each firm i, the component of the State of Nature $\tau_{i}$ enters into its profit function (see below), but is unknown to the firm. Instead, before setting a price or quantity, it - costlessly - receives a noisy signal $y_{i}$ about $\tau_{i}$ as private information: $y_{i}:=\tau_{i}+\eta_{i}$. The random vector $\eta=\left(\eta_{1}, \ldots, \eta_{n}\right)^{\prime}$ is jointly normally distributed with $\mathrm{E}\left(\eta_{i}\right)=0, \operatorname{Var}\left(\eta_{i}\right)=u_{i i} \geq 0$, and $\operatorname{Cov}\left(\eta_{i} \eta_{j}\right)=u_{N} \in\left[0, \min _{i}\left\{u_{i i}\right\}\right]$ for all $i, j(i \neq$ $j)$. Thus the covariance matrix of $\eta$ is $U$ with $u_{11}, \ldots, u_{n n}$ on the main diagonal and $u_{N}$ everywhere else. Furthermore, we assume that $\tau$ and $\eta$ are independent, which implies $\operatorname{Cov}(\mathbf{y})=\mathbf{T}+\mathbf{U}=: \mathbf{P}$.

The variance $u_{i i}$ of the signal error measures the precision of firm i's signal: $u_{i i}=0$ corresponds to $i$ being perfectly informed about $\tau_{i}$ (e.g. its own cost); for $u_{i i}=\infty, y_{i}$ does not convey any information, and a positive $u_{i i}$ implies a noisy signal. Note that the signal precisions may be different for all firms.

We follow Gal-Or (1985) in allowing that the signal errors $\eta_{i}$ be correlated. For example, publicly accessible predictions about business cycles might enter into all $y_{i}$ 's inducing a correlation which has nothing to do with true State of Nature. Hence the private signals may be correlated (i) due to a correlation of the components of the State

\footnotetext{
${ }^{2}$ For convenience, both the random variable and its realizations (hence particular States of Nature) are denoted by $\tau$.
} 
of Nature and (ii) due to correlation of the signal errors. ${ }^{3}$ We assume throughout that the correlation of the $\eta_{i}$ is not greater than the correlation of the $\tau_{i}$. This is stated more precisely in

Assumption COR: $t_{N} u_{i i} \geq t_{H} u_{N} \quad \forall i$.

Let $t_{N} / t_{H}=: \rho_{\tau}$ and $u_{N} / \sqrt{u_{i i} u_{j j}}=: \rho_{\eta}^{i j}$ (for $u_{i i}, u_{j j}>0$ ) denote the correlation coefficients of $\tau$ and $\eta$, respectively. Then COR implies $\rho_{\eta}^{i j} \leq \rho_{\tau}$ for all $i$ and $j$. If the $u_{i i}$ are all equal, the two statements are equivalent. Assumption COR is automatically satisfied for all models of the literature. In the general model this assumption has to be made explicitly; its significance will become clear in the next section.

Information revelation: Firms reveal their private information completely, partially, or not at all to all other firms by means of a signal $\hat{y}_{i}:=y_{i}+\xi_{i}$, where $\xi_{i}$ is normally distributed with zero mean and variance $r_{i}$. The $\xi_{i}$ are independent among each other and from $\boldsymbol{\tau}$ and $\eta$, hence for $\mathbf{r}=\left(r_{1}, \ldots, r_{n}\right)^{\prime}$ and $\hat{\mathbf{y}}=\left(\hat{y}_{1}, \ldots, \hat{y}_{n}\right)^{\prime}$ we have $\operatorname{Cov}(\xi)$ $=\operatorname{diag}(\mathbf{r})$ and $\operatorname{Cov}(\hat{\mathbf{y}})=\mathrm{T}+\mathrm{U}+\operatorname{diag}(\mathbf{r})=: \mathbf{Q}$. The variance $r_{i}$ of the noise added to the true signal $y_{i}$ expresses the revelation behavior of firm $\mathrm{i}$ : for $r_{i}=0, y_{i}$ is completely revealed to the other firms; for $r_{i}=\infty$ a noisy signal with infinite variance is revealed, which is equivalent to concealing private information. For $0<r_{i}<\infty$, private information is revealed partially: the signal $y_{i}$ is distorted by the noise $\xi_{i}$, which reduces the informativeness of $\hat{y}_{i}$ according to the variance $r_{i} \cdot{ }^{5}$ Note that $y_{i}$ cannot be strategically distorted, since $\xi_{i}$ and $y_{i}$ are independent and $\xi_{i}$ has zero mean. Hence apart from random noise, private information is (if at all) revealed truthfully,

${ }^{3}$ For analytical reasons we require that the covariances between the signal errors are the same, which is a limitation of the model if the signal precisions are asymmetric. Thus we may either study the implications of correlated signal errors, assuming equal precisions, or analyze the effects of asymmetric precisions, assuming uncorrelated signal errors.

4 In Gal-Or's (1985) model, however, the conditional correlation of the signal errors for a given State of Nature is nonpositive, an assumption for which Gal-Or does not provide an economic rationale.

${ }^{5}$ Basar/Ho (1974) give information theoretic reasons for calling a signal with a smaller variance a "better" or more informative signal. 
or equivalently, revealed information can be verified at no $\operatorname{cost}^{6}$

Strategies and payoffs: Finally, we turn to the market structure of the model. Demand and cost functions are not explicit elements of the model. Instead, we directly formulate the profit functions. Each firm i controls the variable $s_{i}$, which is either the price of the good produced by $i$ (Bertrand markets) or the quantity supplied (Cournot). The payoff for firm $i$ is given by

$$
\pi_{i}=a_{i}\left(\tau_{i}\right)+\sum_{j \neq i}\left(b_{N}+c_{N} \tau_{i}-\varepsilon s_{i}\right) s_{j}+\left(b_{i i}+c_{H} \tau_{i}-\delta s_{i}\right) s_{i},
$$

where $a_{i}\left(\tau_{i}\right)$ is any function of $\tau_{i}$, and $b_{i i}, b_{N}, c_{H}, c_{N}, \delta$, and $\varepsilon$ are parameters. For reasons to be discussed below, we assume that $\delta>0$ and $\varepsilon \in\left(-\frac{1}{n-1} \delta, \delta\right]$.

The parametric profit function (2.1) suits a large range of standard oligopoly models, in particular, all types discussed in the information sharing literature. This includes Cournot models with a linear demand system and linear or quadratic costs and Bertrand models with a linear demand system and linear costs, both for $\mathrm{n}$ firms producing heterogeneous goods.

To illustrate this point, and since there are no clear-cut economic interpretations of the parameters, we show how the payoff functions of two specific oligopoly models fit into equation $(2.1):^{7}$

(i) We first consider a homogeneous Cournot oligopoly with demand uncertainty. ${ }^{8}$

6 This concept of partial revelation, which is due to Gal-Or (1985), might at first glance seem rather artificial. It might look more natural to think of partial revelation the following way (cf. Li 1985, Vives 1984): assume that the signal $y_{i}$ is the sample mean - i.e. a sufficient statistic - of $n_{i}$ normally distributed single observations $z_{i j}$, i.e. $y_{i}=\left(1 / n_{i}\right) \sum_{j=1}^{n_{i}} z_{i j}$. A subset of these observations (e.g. the first $\left.m_{i}\right)$ is revealed to the other firms by means of sufficient statistic $\hat{y}_{i}=\left(1 / m_{i}\right) \sum_{j=1}^{m_{1}} z_{i j}$, the other observations remain private information. This approach is almost equivalent to the approach described above: since $\hat{y}_{i}=\left(n_{i} / m_{i}\right) y_{i}-\left(1 / m_{i}\right) \sum_{m+1}^{n} z_{i j}$, the revealed signal is, in essence, the received signal plus added noise (the second term), the only difference to the approach described above being the multiplication of $y_{i}$ by $n_{i} / m_{i}$. In particular, increasing the number of revealed observations from 0 to $n_{i}$ corresponds - ignoring integer aspects - to a decrease of the variance of the error term from infinity to 0 .

7 The symbols used in the following examples have nothing to do with those of the general model.

8 This is the most common case of the literature; cf. Ponssard (1979), Novshek/Sonnenschein 
The inverse demand (net of costs) is $P=A+U-B \sum_{j=1}^{n} Q_{j}$, where $\mathrm{P}$ is the price, $Q_{j}$ the supply of firm $j$, and $A$ and $B$ are (deterministic) parameters. $U$ is stochastic with $\mathrm{E}(\mathrm{U})=0$. The profit of firm $\mathrm{i}$ is: $\left(A+U-B \sum_{j=1}^{n} Q_{j}\right) Q_{i}$. Fitting this profit function into the notation of (2.1) yields $s_{i}=Q_{i}, \tau_{i}=U, a_{i}(\cdot)=0, b_{i i}=A, b_{N}=0, c_{H}=$ $1, c_{N}=0$ and $\delta=\varepsilon=B$.

(ii) The second example is an n-firm Bertrand oligopoly with heterogeneous goods and cost uncertainty: ${ }^{9}$ The demand of firm $i$ is $Q_{i}=A-B P_{i}-C \sum_{j=1}^{n} P_{j}$, where for substitute goods $\mathrm{C}$ is negative. The marginal cost is the random variable $U_{i}$ with $\mathrm{E}\left(U_{i}\right)=0$, where we ignore the mean of the marginal cost. The profit then is $(A-$ $\left.B P_{i}-C \sum_{j=1}^{n} P_{j}\right)\left(P_{i}-U_{i}\right)=-A U_{i}+\sum_{j \neq i}\left(C U_{i}-C P_{i}\right) P_{j}+\left(A+B U_{i}-B P_{i}\right) P_{i}$, and for the notation of (2.1) we arrive at: $s_{i}=P_{i}, \tau_{i}=U_{i}, a_{i}\left(\tau_{i}\right)=-A U_{i}, b_{i i}=A, b_{N}=$ $0, c_{H}=\delta=B$, and $c_{N}=\varepsilon=D_{N}$.

We first note that the profit of firm $j$ is affected by the State-of-Nature-components $\tau_{j}(j \neq i)$ only indirectly through the strategies $s_{j}$ of the other players. Secondly, the assumption that $\tau$ has zero mean does not impose any restrictions.

For all models with demand uncertainty (Cournot or Bertrand), $c_{H}$ equals 1 , and for Cournot models with cost uncertainty, $c_{H}$ equals -1 . In all these cases, $c_{N}$ equals zero. Hence (i) for Cournot competition, $c_{H}$ indicates the source of uncertainty, and (ii) only in the case of a Bertrand market with cost uncertainty, $c_{N}$ will take a nonzero value, the importance of which will be seen in later sections.

Although not modelled explicitly, the oligopoly model has an underiying linear demand system of the form $p=a-D$ s for a Cournot market or $q=a-D$ s for $a$ Bertrand market, where $\mathbf{D}$ is an n-matrix with $\delta$ on the main diagonal and $\varepsilon$ everywhere else, $\mathrm{p}$ and $\mathrm{q}$ (not used for later reference) denote the vectors of prices and quantities, respectively, $s=\left(s_{1}, \ldots, s_{n}\right)^{\prime} \in I R^{n}$ is the vector of strategies (prices or quantities), and $\mathrm{a} \in \mathbb{R}^{\mathrm{n}}$ is some parameter vector. ${ }^{10}$ Such a demand system can be derived as the (1982), Clarke (1983), Vives (1984), Gal-Or (1985), Li (1985).

${ }^{9}$ Bertrand oligopolies with more than two firms have so far not been considered in the literature.

10 Obviously, for economic reasons we would restrict the strategy space to the nonnegative orthant of $I R^{n}$. However, when deriving the equilibrium stragies of the oligopoly game it is convenient to 
first-order condition of a representative consumer's maximization of an appropriately defined utility function and is the simplest way of incorporating product differentiation into the model (cf. Vives 1984, Sakai/Yamato 1989). ${ }^{11}$ That the derived demand system is indeed the solution of a maximization problem requires that the matrix $D$ (or $\mathbf{D}^{-1}$, respectively) be positive definite, which leads to the restriction on $\varepsilon$ and $\delta$ stated above.

Having discussed the elements of the model, we can now formulate the explicit game(s) that will be analyzed. The model consists of the following stages:

(i) Firms decide on their revelation behavior by setting $r_{i}$. We will consider two variants: (a) firms enter into a contract specifying that information shall be revealed completely or not at all, i.e. $r_{i}=0 \forall i$ or $r_{i}=\infty \forall i$; (b) firms set the $r_{i}$ 's simultaneously, where we exclude partial revelation but allow asymmetric behavior, i.e. $r_{i} \in[0, \infty] \forall i$.

(ii) The State of Nature $\tau$ is determined randomly. The players know the distribution of $\tau$ but not its realization.

(iii) Each firm $\mathrm{i}$ receives a private signal $y_{i}$. The distribution of $\mathbf{y}$ is common knowledge.

(iv) $y_{i}$ is revealed completely, partially, or not at all to all other firms by means of $\hat{y}_{i}$. The revelation behavior is given by $r_{i}$, and $\mathbf{r}$ is known to all firms.

(v) Firms play the oligopoly game, i.e. each firm i sets the price/quantity $s_{i}$ as a function of the information $z_{i}:=\left(y_{i}, \hat{y}^{\prime}\right)^{\prime}$ accessible to firm $\mathbf{i}$.

drop this restriction and ignore the possibility that for extreme realizations of the random variables the nonnegativity conditions might actually bind.

${ }^{11}$ Let the representative buyer have the utility function $U\left(q_{0}, \mathbf{q}\right)=q_{0}+\tilde{\mathbf{a}}^{\prime} \mathbf{q}-\frac{1}{2} \hat{\mathbf{q}}^{\prime} \hat{\mathbf{D}} \hat{\mathbf{q}}$, where $q_{0}$ is the numeraire good and utility is measured in units of the numeraire. If $\hat{\mathrm{D}}$ has the structure $\hat{\mathrm{D}}=\hat{\delta} \mathrm{I}+\hat{\varepsilon} \overline{\mathrm{I}}$, then utility maximization under a budget constraint leads to the first order condition $\mathbf{p}=\mathbf{\mathbf { a }}-\hat{\mathbf{D}} \mathbf{q}$, which is a linear demand system of the above-mentioned structure: for Cournot models, $D=\bar{D}$ and $s=q$, and for Bertrand $D=\hat{D}^{-1}$ and $s=p$. The goods are substitutes if $\hat{\varepsilon}>0$ and complements if $\hat{\varepsilon}<0$. 
Discussion of the model:

(i) The State of Nature and the structure of private information comprise the exogenous information structure of the model. In Section 4, we will focus on some special cases which the literature has restricted attention to. The first is the case of a "Common Value", where according to the usual modelling the State of Nature is a skalar entering into all firms' profits. In our general model, we can equivalently assume that the n (identically distributed) components of the State of Nature are perfectly correlated, since then all $\tau_{i}$ are equal with probability one. For statistical decisions there is no difference between this specification and the usual common-value assumption. We refer to this case as

Assumption CV (Common Value): $t_{N}=t_{H}=: t$

All other cases, in which the State of Nature is a nondegenerate n-vector, have been referred to as "private-value" models. However, here we will restrict this notion to the case where the components of the State of Nature are uncorrelated:

Assumption $P V$ (Private Values): $t_{N}=u_{N}=0$,

where setting $u_{N}$ to zero (uncorrelated signal errors) follows from COR. In fact, the work of Gal-Or (1986) is the only one in which assumption PV is made. In most of the other "private-value" models, any correlation between the State-of-Nature components is allowed for. But it is additionally assumed that firms receive signals without noise, i.e. acquire perfect knowledge about their "own" $\tau_{i}$. We refer to this case as Assumption PS (Perfect Signals): $u_{i i}=u_{N}=0 \quad \forall i$.

Hence in this case, $\eta$ degenerates to a zero distribution. Our separation of models classified as "private-value" models in the literature into two categories has two reasons: First, it seems more appropriate to refer to a "common value" and "private values" as limit cases of the covariance of the $\tau_{i}, t_{N}$, lying between 0 and $t_{H}$ rather than speaking of a common value for $t_{N}=t_{H}$ and of private values for $t_{N} \in\left[0, t_{H I}\right)$. Second, only by taking the impact of signal noise into account the apparent inconsistency pointed 
out in the introduction between the results in common-value models and the results in "private-value" models where the correlation of the State-of-Nature components approaches unity can be explained: The existence or nonexistence of signal noise is the only remaining difference between these types of models. The role of signal noise has not received any attention in the previous literature.

(ii) Assuming normal random variables ensures that all conditional expectations are affine functions of the given information variables. On the other hand, the support is the entire set of real numbers, thus in principle negative demand intercepts or costs can occur. This problem is usualiy regarded as negligible, since variances can always be set so as to make the probability of undesirable signs arbitrarily small. Alternatively, $\mathrm{Li}$ (1985) and Shapiro (1986) have allowed for any distributions possessing the linear expectations property, thus admitting distributions with a compact support.

(iii) The assumption that firms must make an ex-ante commitment as to their revelation behavior is problematic, since for some signals there is an incentive to deviate from the previously chosen revelation behavior. In particular, it has been argued that while it seems reasonable to allow for commitments always to reveal private information, it is much less reasonable to consider commitments never to reveal private information, since information, once acquired, would would always be passed on to other firms if this was deemed profitable (Okuno-Fujiwara, Postlewaite and Suzumura 1990).

On the other hand, the dichotomy between deciding on the revelation behavior before or after private information is received is imposed by the static structure of the model; and assuming - exclusively - ex-post decisions does not seem more plausible in our context if the two-stage model is interpreted as a reduced form (or metaphor) of a multiperiod repeated interaction, which is a common procedure in economic theory. Then the ex-post/ex-ante distinction amounts to a difference between myopic rentseeking and long-run profit maximization. In particular, it seems reasonable to assume that a firm can resist the short-run temptation to reveal private information if this is in its long-run interest, since revelation would immediately give rise to the "unravel- 
ling process" driving many ex-post-revelation models where the agents end up always revealing information. ${ }^{12}$

(iv) Revealed information is accessible to all other firms, whether these reveal or not. Such nonexclusionary disclosure of information is assumed in almost all works. Kirby (1988) has studied information sharing agreements where nonrevealing firms are excluded from the pooled information. This issue will be taken up again in Section 4.4.

(v) By (2.1), $\partial^{2} \pi_{i} / \partial s_{i} \partial s_{j}=-\varepsilon$. Hence the sign of $\varepsilon$ indicates whether we are dealing with strategic substitutes or complements in the sense of Bulow, Geanakoplos and Klemperer (1985). For $\varepsilon>0$ (e.g. Cournot with substitute goods or Bertrand with complements) we have a game of strategic substitutes, meaning that reaction curves are downward-sloping. For $\varepsilon<0$ (Bertrand with substitute goods or Cournot with complements) we have strategic complements, with upward-sloping reaction curves.

(vi) Firms are assumed to be risk-neutral, thus they maximize their expected profits. Information sharing with risk-averse duopolists is analyzed by Hviid (1989); however, taking risk-aversion into account significantly complicates the analysis and requires restriction to very simple oligopoly models.

Almost all models of the literature are special cases of the model developed here, resulting by appropriately specifying the parameters. ${ }^{13}$ These specifications are shown in Table 2, where $\iota:=(1, \ldots, 1)^{\prime}$. Note in particular that all models belong to one of the three classes CV, PV, and PS introduced above.

12 Okuno-Fujiwara, Postlewaite and Suzumura (1990) analyze models with ex-post decisions at a general level.

13 Shapiro (1986) and $\mathrm{Li}$ (1985) make slightly more general assumptions as to the distributions of the random variables; Shapiro (1986) considers (in our notation) $\tau_{i}$ 's with different variances and the same correlation; Sakai's (1986) perfect-signal duopoly model allows arbitrary matrices $D$ and $T$. The model of Novshek/Sonnenschein (1982) does not fit into our framework except for the uninteresting case of a common value and perfect signals (cf. the discussion in Clarke 1983), and Hviid (1989) assumes risk-averse duopolists. These are the only exceptions. 
Table 2: Previous models as special cases of the general model

\begin{tabular}{|l||r|r|r|r|r|r|l|l|l||}
\hline \hline model & $n$ & $a_{i}\left(\tau_{i}\right)$ & $\varepsilon$ & $c_{H}$ & $c_{N}$ & $b_{i i}$ & $b_{N}$ & $\mathrm{~T}, \mathrm{U}$ & $\mathbf{r}$ \\
\hline \hline Clarke (1983) & $n$ & 0 & $\delta$ & 1 & 0 & equal & 0 & $\mathrm{CV}, \rho_{\eta}=0$ & $\mathbf{r} \in\{0, \infty \iota\}$ \\
Fried (1984) & 2 & 0 & $\delta$ & 1 & 0 & diff. & 0 & $\mathrm{PS}$ & $r_{i} \in\{0, \infty\}$ \\
Vives (1984) & 2 & 0 & any & 1 & 0 & equal & 0 & $\mathrm{CV}, \rho_{\eta}=0$ & $r_{i} \in[0, \infty]$ \\
Gal-Or (1985) (1) & $n$ & 0 & $\delta$ & 1 & 0 & equal & 0 & $\mathrm{CV}, u_{N}=-t_{N}$ & $r_{i} \in[0, \infty]$ \\
Gal-Or (1985) (2) & 2 & 0 & $\delta$ & 1 & 0 & equal & 0 & $\mathrm{CV}, \rho_{\eta} \leq 0$ & $r_{i} \in[0, \infty]$ \\
Li (1985) (1) & $n$ & 0 & $\delta$ & 1 & 0 & equal & 0 & $\mathrm{CV}, \rho_{\eta}=0$ & $r_{i} \in[0, \infty]$ \\
Li (1985) (2) & $n$ & 0 & $\delta$ & -1 & 0 & equal & 0 & $\mathrm{PS}$ & $r_{i} \in[0, \infty]$ \\
Gal-Or (1986) (1) & 2 & 0 & any & -1 & 0 & equal & 0 & $\mathrm{PV}$ & $r_{i} \in[0, \infty]$ \\
Gal-Or (1986) (2) & 2 & $-b_{i i} \tau_{i}$ & any & $\delta$ & $\varepsilon$ & equal & 0 & $\mathrm{PV}$ & $r_{i} \in[0, \infty]$ \\
Shapiro (1986) & $n$ & 0 & $\delta$ & -1 & 0 & equal & 0 & $\mathrm{PS}$ & $\mathbf{r} \in\{0, \infty \iota\}$ \\
Sakai (1986) & 2 & 0 & any & 1 & 0 & diff. & 0 & $\mathrm{PS}$ & $r_{i} \in\{0, \infty\}$ \\
Kirby (1988) & $n$ & 0 & any & 1 & 0 & equal & 0 & $\mathrm{CV}, \rho_{\pi}=0$ & $r_{i} \in\{0, \infty\}$ \\
Sakai/ & & & & & & & & \\
Yamato (1989) & $n$ & 0 & any & -1 & 0 & equal & 0 & $\mathrm{PS}$ & $\mathbf{r} \in\{0, \infty \iota\}$ \\
\hline \hline
\end{tabular}

\section{Nash Equilibrium of the Oligopoly Game}

In this section, we derive the Bayesian Nash equilibrium of the oligopoly game. At this last stage, the revelation behavior $\mathbf{r}=\left(r_{1}, \ldots, r_{n}\right)^{\prime}$ is known to all firms, and each firm i has information $\mathbf{z}_{\mathbf{i}}=\left(y_{i}, \hat{\mathbf{y}}^{\prime}\right)^{\prime}$. The Bayesian Nash equilibrium $\mathbf{s}^{*}$ of this subgame is characterized by

$$
s_{i}^{*}\left(\mathbf{z}_{\mathbf{i}}\right)=\arg \max _{s_{i} \in I R^{n}} E_{\tau, \eta_{-i}}\left[\pi_{i}\left(s_{i}, \mathbf{s}_{-\mathbf{i}}^{*} \mid \mathbf{z}_{\mathbf{i}}\right)\right] \quad(i=1, \ldots, n),
$$

leading to the reaction functions

$$
s_{\mathbf{i}}=\frac{1}{2 \delta}\left[b_{i i}+c_{H} E\left(\tau_{i} \mid \mathbf{z}_{\mathbf{i}}\right)-\varepsilon \sum_{j \neq i} E\left(s_{j} \mid \mathbf{z}_{\mathbf{i}}\right)\right] \quad(i=1, \ldots, n),
$$


where expectations are formed over all random variables unknown at this stage, i.e. the State of Nature and the signal errors $\eta_{-i}$ of the rival firms. Since the expectations $E\left(s_{j} \mid \mathbf{z}_{\mathbf{i}}\right)$ cannot be evaluated unless at least the functional form of the $s_{j}\left(\mathbf{z}_{\mathbf{j}}\right)$ is known, we have to proceed in two steps to compute the equilibrium: First, we prove existence and uniqueness of an equilibrium with strategies $s_{i}$ that are affine functions of $\mathbf{z}_{\mathbf{j}}$. In the second step, the coefficients of these functions are computed.

Define $f(s):=\sum_{i=1}^{n}\left[b_{i i}+c_{H} \tau_{i}-(\varepsilon / 2) \sum_{j \neq i} s_{j}-\delta s_{i}\right] s_{i}$. Suppose that every agent $\mathrm{i}$ controls $s_{i} \in I R$, has knowledge of $z_{i}$, and seeks to maximize the expected value of $f$, where the information structure is the same as described in the last section. Then we are dealing with a team decision problem in the sense of Radner (1962), i.e. a number of agents controlling different action variables and with different access to information, but with a common objective function. By construction of $f$, the solution of the team decision problem corresponds to the Nash equilibrium of the oligopoly game. We then use this equivalence result to prove existence, uniqueness and linearity of the Nash equilibrium by applying a result from team theory:

Proposition 3.1 There exists a unique Nash equilibrium of the oligopoly game for given information vectors $\mathbf{z}_{\mathbf{i}} \quad(i=1, \ldots, n)$. The equilibrium strategies $s_{i}\left(\mathbf{z}_{\mathbf{i}}\right)$ are affine in $\mathrm{z}_{\mathrm{i}}$, i.e. for all $i$, there exist $\alpha_{i}, \beta_{i} \in \mathbb{R}$ and $\gamma_{i} \in \mathbb{R}^{n}$, such that $s_{i}=\alpha_{i}+\beta_{i} y_{i}+\gamma_{i}^{\prime} \hat{\mathbf{y}}$.

Proof: First of all, maximization of the expected value of $f$ requires $s_{i}^{*}\left(z_{i}\right)=$ $\operatorname{argmax}_{s_{i}} E\left[f\left(\mathrm{~s}^{*}\right) \mid z_{i}\right] \quad \forall i$, which leads to the first-order conditions (3.1). Similarly, all higher order conditions for the Nash equilibrium and the solution of the team decision problem are identical. Thus given the unbounded strategy space, a vector of functions $s_{\mathbf{i}}^{*}\left(\mathbf{z}_{\mathbf{i}}\right) \quad(i=1, \ldots, n)$ is a solution of the team decision problem defined by $f$ if and only if it is a Nash equilibrium of the oligopoly game. Then the main result is immediately obtained by application of Theorem 5 of Radner (1962) to the team function $f$ defined above. The applicability of the theorem requires that (i) $f$ is quadratic in s, (ii) the matrix $\Psi:=(\delta-\varepsilon / 2) \mathbf{I}+(\varepsilon / 2) \iota \iota^{\prime}$ is deterministic, (iii) $\Psi$ is positive definite, (iv) the information vectors and the first-best solution of the team decision problem 
$(1 / 2) \Psi^{-1}\left[\left(b_{11}, \ldots, b_{n n}\right)^{\prime}+c_{H} \tau\right]$ are jointly normally distributed. Positive definiteness of $\Psi$ follows from the assumption that $\mathrm{D}$ is positive definite, and all other conditions are obviously satisfied. ${ }^{14}$

Having established linearity of the equilibrium strategies, we now compute the coefficients $\alpha_{i}, \beta_{i}$, and $\gamma_{i}$. To evaluate the first-order conditions (3.1), we first compute the conditional expectations $E\left(\tau_{i} \mid \mathbf{z}_{\mathbf{i}}\right)$ and $E\left(y_{j} \mid \mathbf{z}_{\mathbf{i}}\right)$.

Define $p_{i i}:=t_{H}+u_{i i} \forall i$ and $p_{N}:=t_{N}+u_{N}$. Thus $p_{i i}$ and $p_{N}$ are the variances and covariances of the the signals $y_{i}$, respectively. Furthermore, define $m_{i}:=p_{i i}-p_{N}+$ $r_{i}, \vec{m}_{i}:=1 / m_{i}$, and $\overline{\mathbf{m}}:=\left(\bar{m}_{1}, \ldots, \bar{m}_{n}\right)^{\prime}$. Finally, let $\mathbf{e}_{\mathbf{i}}$ denote the $\mathbf{i}$-th unit vector.

Proposition 3.2 For given $\mathrm{z}_{\mathrm{i}}$, the conditional expectations for $\tau_{i}$ and $y_{j}$ are

$$
\begin{aligned}
& E\left(\tau_{i} \mid \mathbf{z}_{\mathbf{i}}\right)=g_{i} y_{i}+\hat{\mathbf{g}}_{\mathbf{i}}^{\prime} \hat{\mathbf{y}} \quad \text { and } \quad E\left(s_{j} \mid \mathbf{z}_{\mathbf{i}}\right)=h_{i j} y_{i}+\hat{\mathbf{h}}_{\mathbf{i j}}^{\prime} \hat{\mathbf{y}} \quad(j \neq i), \quad \text { where } \\
& g_{i}=\frac{\theta_{i}}{D_{i}} \quad \hat{\mathrm{g}}_{\mathbf{i}}=\frac{1}{D_{i}}\left(t_{N} p_{i \mathrm{i}}-t_{H} p_{N}\right)\left(\overline{\mathbf{m}}-\bar{m}_{\mathbf{i}} \mathbf{e}_{\mathbf{i}}\right) \\
& h_{i j}=\frac{p_{N} r_{i} \bar{m}_{j}}{D_{i}} \quad \bar{h}_{i j}=\left(p_{j j}-p_{N}\right) \bar{m}_{j} \mathbf{e}_{j}+h_{i j}\left(p_{i i}-p_{N}\right)\left(\overline{\mathbf{m}}-\bar{m}_{i} \mathbf{e}_{\mathbf{i}}\right) \text {, and } \\
& M=\sum_{j=1}^{n} \bar{m}_{i} \quad D_{i}=p_{i i}+p_{N}\left(p_{i i}-p_{N}\right)\left(M-\bar{m}_{i}\right) \\
& \theta_{i}=t_{H}+p_{N}\left(t_{H}-t_{N}\right)\left(M-\bar{m}_{i}\right) \text {. }
\end{aligned}
$$

For the proofs of this proposition and all subsequent lemmas and propositions, see Appendix A.

Setting $r_{i}=\infty$ for all $\mathrm{i}$ implies $\overline{\mathrm{m}}=\mathbf{0}$ and $\hat{\mathbf{g}}_{\mathbf{i}}=\hat{\mathbf{h}}_{\mathbf{i}}=\mathbf{0}$ for all $\mathbf{i}$. Thus no use is made of the revealed signals $\hat{\mathbf{y}}$, which is equivalent to a situation without information sharing. ${ }^{15}$ Similarly, for $u_{i i}=\infty, g_{i}=h_{i j}=0$, i.e. player $i$ does not make use of $y_{i}$, since it does not convey any useful information. This corresponds to player i not receiving any private information.

14 See Basar/Ho (1974) for a similar application of Radner's theorem to a duopoly model and Vives (1988) for an application to competitive markets. Games for which common objective functions (like $f$ in our case) can be constructed are studied in more detail by Monderer and Shapley (1991), who call such games "potential games".

15 For infinite variances of $r_{i}$ or $u_{i j}$ we sometirnes implicitly form limits to apply expressions of the kind of Proposition 3.2. For example, "for $r_{i}=\infty, r_{i} \bar{m}_{i}=1$ is meant in the sense that $\lim _{r_{i} \rightarrow \infty} r_{i} \bar{m}_{i}=1$. 
The subtraction of $\bar{m}_{i} e_{i}$ in the expressions for $\hat{\mathbf{g}}_{i}$ and $\hat{\mathbf{h}}_{\mathbf{i}}$ implies that player $i$ never makes use of $\bar{y}_{i}$ when forming expectations about $\tau_{i}$ and $y_{j}$. This is intuitively clear because $i$ already knows the "better" signal $y_{i}$. Moreover, the subtraction of $\tilde{m}_{i}$ in the expressions for $g_{i}$ and $h_{i j}$ implies that the expectations for $\tau_{i}$ and $y_{j}$ do not depend on the own revelation behavior.

The expression for $\hat{\mathbf{g}}_{\mathbf{i}}$ makes the significance of assumption COR clear: since $t_{N} p_{i i}-$ $t_{H} p_{N}=t_{N} u_{i i}-t_{H} u_{N}, \mathrm{COR}$ implies that the components of $\hat{\mathbf{g}}_{\mathrm{i}}$ are nonnegative, which in turn ensures that correlations of $y_{i}$ and $y_{j}$ are attributed to a correlation of the underlying State-of-Nature components rather than to a correlation of the signal errors.

Substituting $E\left(s_{j} \mid \mathbf{z}_{\mathbf{i}}\right)=\alpha_{j}+\beta_{j} E\left(y_{j} \mid \mathbf{z}_{\mathbf{i}}\right)+\gamma_{j}^{\prime} \hat{\mathbf{y}}$ and the expressions from Proposition 3.2 in (3.1) yields

$$
s_{i}\left(\mathbf{z}_{\mathbf{i}}\right)=\frac{1}{2 \delta}\left[\left(b_{i i}-\varepsilon \sum_{j \neq i} \alpha_{j}\right)+\left(c_{H} g_{i}-\varepsilon \sum_{j \neq i} \beta_{j} h_{i j}\right) y_{i}+\left(c_{H} \hat{\mathbf{g}}_{i}^{\prime}-\varepsilon \sum_{j \neq i} \beta_{j} \hat{\mathrm{h}}_{\mathrm{ij}}^{\prime}+\gamma_{j}^{\prime}\right) \hat{\mathbf{y}}\right]
$$

On the other hand, $s_{i}=\alpha_{i}+\beta_{i} y_{i}+\gamma_{i}^{\prime} \hat{y}$. Identification of these coefficients with the corresponding terms in (3.2) leads to the main result of this section:

Proposition 3.3 In the Bayesian Nash equilibrium of the oligopoly game each firm $i$ $(i=1, \ldots, n)$ has the strategy $s_{i}\left(\mathbf{z}_{\mathbf{i}}\right)=\alpha_{i}+\beta_{i} y_{i}+\gamma_{i}^{\prime} \hat{\mathbf{y}}$, where

$$
\begin{aligned}
\alpha_{i}= & \frac{1}{\tilde{d}}\left(b_{i i}-\frac{\varepsilon}{\hat{d}} \sum_{j=1}^{n} b_{i i}\right), \\
\beta_{i}= & \frac{c_{H}}{v_{i}}\left(0_{i}-\varepsilon p_{N} \frac{\sum_{j=1}^{n} \frac{r_{i} \bar{m}_{i} \theta_{i}}{v_{i}}}{1+\varepsilon p_{N} \sum_{j=1}^{n} \frac{r_{i} \bar{m}_{i}}{v_{i}}}\right) \quad \text { and } \\
\gamma_{i}= & \frac{2 \delta}{\hat{d}}\left[\left(p_{i i}-p_{N}\right) \beta_{i}-\frac{\varepsilon}{\hat{d}} \sum_{j=1}^{n}\left(p_{j j}-p_{N}\right) \beta_{j}-\frac{c_{H}\left(t_{H}-t_{N}\right)}{\hat{d}}\right] \overline{\mathbf{m}} \\
& -\left[\left(p_{i i}-p_{N}\right) \beta_{i}-\frac{c_{H}\left(t_{H}-t_{N}\right)}{\tilde{d}}\right] \bar{m}_{i} \mathbf{e}_{\mathbf{i}}, \quad \text { and } \\
\tilde{d}= & 2 \delta-\varepsilon, \quad \hat{d}=2 \delta+(n-1) \varepsilon, \quad v_{i}=2 \delta D_{i}-\varepsilon p_{N} r_{i} \bar{m}_{i}
\end{aligned}
$$

The equilibrium strategies of the models of other works result as corollaries of Proposition 3.3: this applies for Ponssard (1979), Clarke (1983), Fried (1984), Vives (1984; 
Propositions 2, 2a ); Gal-Or (1985, Theorems 1 and 2), Gal-Or (1986, Lemmas 1 and 2), Shapiro (1986), Li (1985, first model, Proposition 1), Kirby (1988), Sakai (1986, with the caveat of footrote 13) and Sakai/Yamato (1989).

$$
E(\boldsymbol{\tau})=E(\boldsymbol{\eta})=E(\boldsymbol{\xi})=0 \text { implies } E\left(s_{i}\right)=\alpha_{i} \text {. Hence the expected value of } s_{i}
$$
is invariant with respect to changes in $\mathbf{T}, \mathbf{P}$, or $\mathbf{r}$; in particular it is not affected by information sharing.

The vector $\gamma_{i}$ consists of a general term which treats all $\hat{y}_{j}$ equally (but taking $r_{i}$ into account), and a correction term applied to $\hat{y}_{i}$. These terms do not cancel each other, hence $s_{i}$ depends on $\hat{y}_{i}$ even though firm $i$ knows the true $y_{i}$. This is due to the fact that although $\hat{y}_{i}$ is not needed for expectations about $\tau_{i}$ or $y_{j}, \hat{y}_{i}$ enters into $E\left(s_{j} \mid \mathrm{z}_{\mathfrak{i}}\right)(j \neq i)$ since $\mathrm{j}$ makes use of $\hat{y}_{i}$.

Setting $r_{i}=\infty$ for all $\mathrm{i}$ implies $\overline{\mathbf{m}}=\mathbf{0}$ and hence $\boldsymbol{\gamma}_{i}=\mathbf{0}$ for all $\mathrm{i}$. This corresponds to a situation without information sharing, i.e. the strategies for the no-sharing case follow as a special case from Proposition 3.3.

We now derive the expected profits for the equilibrium of the oligopoly game, where expectations are formed for unknown $z_{\mathfrak{i}}$ (i.e., before firms receive private information) but known revelation behavior $r$. According to (2.1), firm i's profit is

$$
\pi_{i}=a_{i}\left(\tau_{i}\right)+\left(b_{N}+c_{N} \tau_{i}\right) \sum_{j \neq i} s_{j}+\left[b_{i i}+c_{H} \tau_{i}-\varepsilon \sum_{j \neq i} s_{j}-\delta s_{i}\right] s_{i}
$$

We take expectations of (3.3) making use of the fact that $i$ knows $\mathbf{z}_{\mathbf{i}}$ when she determines $s_{i}: \mathrm{E}\left(\pi_{i}(\mathrm{~s})\right)$ equals

$$
\mathrm{E}\left(a_{i}\left(\tau_{i}\right)\right)+E\left[\left(b_{N}+c_{N} \tau_{i}\right) \sum_{j \neq i} s_{j}\right]+E_{y_{i}, \hat{y}}\left[E\left(b_{i i}+c_{H} \tau_{i}-\varepsilon \sum_{j \neq i} s_{j}-\delta s_{i} \mid \mathbf{z}_{\mathbf{i}}\right) s_{i}\right] .
$$

By (3.1), the last term in (3.4) reduces to $\delta E\left(s_{i}^{2}\right)$, since $s_{i}$ is an equilibrium strategy; and $\mathrm{E}\left(s_{i}^{2}\right)=E^{2}\left(s_{i}\right)+\operatorname{Var}\left(s_{i}\right)=\alpha_{i}^{2}+\operatorname{Var}\left(s_{i}\right)$.

For the second term in (3.4) we get

$$
E\left[\left(b_{N}+c_{N} \tau_{i}\right) \sum_{j \neq i} s_{j}\right]=\sum_{j \neq i}\left(b_{N} \alpha_{j}+c_{N} \beta_{j} t_{N}+c_{N} \gamma_{j}^{\prime} t_{i}\right)
$$


where the expectations are taken from (A.4), and $t_{\mathbf{i}}$ is the $\mathrm{i}$-th column vector of $\mathbf{T}$. Thus the expected profits are $E\left(\pi_{i}(\mathrm{~s})\right)=E\left(\pi_{i}^{C}(\mathrm{~s})\right)+E\left(\pi_{i}^{M}(\mathrm{~s})\right)+E\left(\pi_{i}^{A}(\mathrm{~s})\right)$, where $E\left(\pi_{i}^{C}(\mathbf{s})\right):=E\left(a_{i}\left(\tau_{i}\right)\right)+\delta \alpha_{i}^{2}+b_{N} \sum_{j \neq i} \alpha_{j}, E\left(\pi_{i}^{M}(\mathbf{s})\right):=\delta \operatorname{Var}\left(s_{i}\right)$, and $E\left(\pi_{i}^{A}(\mathrm{~s})\right):=$ $c_{N} \sum_{j \neq i}\left(t_{N} \beta_{j}+\gamma_{j}^{\prime} t_{i}\right)$. In the following analyses we are chiefly concerned with differences of expected profits for different situations of information revelation behavior. Since $E\left(\pi_{i}^{C}\right)$ does not depend on $\mathbf{P}$ or $\mathbf{r}$ it cancels out in these differences.

Moreover, for Cournot markets and for Bertrand markets with demand uncertainty, $c_{N}=0$ and hence $E\left(\pi_{i}^{A}(s)\right)=0$. For most of the following sections we restrict the analysis to these cases. Only section 4.6 is devoted to the remaining case, Bertrand markets with cost uncertainty. ${ }^{16}$

Thus the duality between Cournot markets with substitutes and Bertrand markets with complements sometimes emphasized in the literature (Vives 1984, Sakai 1986) only applies to markets with demand uncertainty, whereas with cost uncertainty the profit functions for Cournot and Bertrand markets are structurally different.

\section{The incentives to share information}

The incentives for firms to share private information have been the focus of research on information sharing. As noted by several authors, these incentives are largely determined by the change in the correlation of strategies induced by the pooling of information. However, it has not been treated analytically how this correlation is actually affected in different settings. Sections 4.1 and 4.2 address this question. Then we study the two approaches to the determination of revelation behavior introduced in section 2: First, we analyze the incentives to completely pool information, compared with no pooling. Alternatively, we derive the equilibrium of the two-stage game where firms first independently decide on their revelation behavior. The results for both approaches are discussed in 4.5 , where we make use of the results of 4.1 and 4.2 . Finally, we turn to

\footnotetext{
${ }^{16}$ Note that the second model of Gal-Or (1986) is the only Bertrand model with cost uncertainty in the literature.
} 
the case excluded for most of this paper, Bertrand markets with cost uncertainty.

For the rest of the paper we assume that $b_{i i}=b_{H}$ for all $i$. For most applications, this means that the firms have the same expected demand intercepts and marginal costs. Moreover, except for section 4.6 we henceforth assume that $c_{N}=0$, which implies $\pi_{i}^{A}=0 \forall i$.

\subsection{No-sharing case}

Complete concealing of private information corresponds to assuming that $r_{i}=\infty$ for all $i$, which implies hence $\gamma_{i}=0 \quad \forall i$. For the parameters of Proposition 3.4 we have: $\theta_{i}=t_{H}, \quad r_{i} \bar{m}_{i}=1$, and $v_{i}=2 \delta p_{i i}-\varepsilon p_{N}$. From Proposition 3.4 we obtain

$$
\beta_{i}=\frac{c_{H}}{v_{i}}\left(t_{H}-\frac{\varepsilon p_{N} t_{H} \sum_{j=1}^{n}\left(1 / v_{i}\right)}{1+\varepsilon p_{N} \sum_{j=1}^{n}\left(1 / v_{i}\right)}\right)=\frac{c_{H} t_{H}}{2 \delta p_{i i}-\varepsilon p_{N} \sum_{j \neq i} v_{i} / v_{j}}
$$

Without information sharing,

$$
\operatorname{Var}\left(s_{i}\right)=p_{i i} \beta_{i}^{2} \text { and } \operatorname{Cov}\left(s_{i} s_{j}\right)=\beta_{i} \beta_{j} E\left(y_{i} y_{j}\right)=p_{N} \beta_{i} \beta_{j}(j \neq i),
$$

hence for the correlation of these strategies $\rho_{s}^{i j}$ we have $\rho_{j}^{i j}=p_{N} / \sqrt{p_{i i} p_{j j}}$, or if $p_{i i}:=$ $p_{H} \forall i: \quad \rho_{s}=p_{N} / p_{H}$. Thus the correlation of $s_{i}$ and $s_{j}$ equals the correlation of the private signals $y_{i}$ and $y_{j}$. Without sharing their private signals, players are not able to discriminate between the underlying State of Nature and the signal errors; therefore the correlation of the strategies does not depend on how the parameters of $T$ and $U$

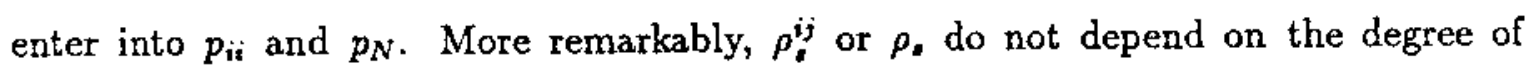
product differentiation expressed by $\varepsilon$ and $\delta$.

Next, we investigate how strategies and profits are influenced by the precision and correlation of the signals. The expected profit of firm $i$ is ${ }^{17}$

$$
E\left(\pi_{i}^{C}\right)+\delta \operatorname{Var}\left(s_{i}\right)
$$

and changes in $\mathbf{T}$ or $\mathbf{P}$ only affect $\operatorname{Var}\left(s_{i}\right)=p_{i i} \beta_{i}^{2}$. We frequently use the notation $a \sim b$ to denote $\operatorname{sign}(a)=\operatorname{sign}(b)$.

${ }^{17}$ The shorthand notation $E\left(\pi_{i}\right)$ refers to the expected profits for equilibrium strategies. 
Lemma 4.1 $\beta_{i} \sim c_{H}$.

This confirms the intuition that the direction of response to a private signal is determined by the source of uncertainty (demand or cost).

Proposition 4.1 ${ }^{18}$ Without information sharing,
(a) $\frac{\partial \beta_{i}}{\partial p_{i i}} \sim-c_{H}$,
(b) $\frac{\partial E\left(\pi_{i}\right)}{\partial p_{i i}}<0$,
(c) $\frac{\partial \beta_{i}}{\partial p_{j j}} \sim c_{H} \varepsilon$
(d) $\frac{\partial E\left(\pi_{i}\right)}{\partial p_{j j}} \sim \varepsilon$.

Part (a) states that a more precise signal implies that the absolute value of $\beta_{i}$ (the sign of which is determined by $c_{H}$ ) increases; hence $\mathrm{i}$ reacts more sensitively to her own signal, which by (4.3) is profitable (part b). According to (c) and (d), a more precise signal of an other firm leads to a decrease of the absolute value of $\beta_{i}$ and of $i$ 's expected profit for strategic substitutes, and to an increase for strategic complements. While (a) and (b) are intuitively clear, the role of strategic substitutes and complements in (c) and (d) is not that obvious; we postpone the dicussion.

For the rest of the paper, we assume that the private signals have equal precisions, i.e. $p_{i i}=p_{H} \forall i$. Then (4.1) implies

$$
\beta_{i}=\beta=\frac{c_{H} t_{H}}{2 \delta p_{H}+(n-1) \varepsilon p_{N}}=\frac{c_{H} t_{H}}{p_{H}\left[2 \delta+(n-1) \varepsilon \rho_{y}\right]},
$$

where $\rho_{y}=p_{N} / p_{H}$ is the correlation of the signals. From $E\left(\pi_{i}(s)\right)=p_{H} \beta^{2}$ we immediately obtain (without proof)

Proposition 4.2 In the completely symmetric model without information sharing,

$$
\text { (a) }\left.\frac{\partial E\left(\pi_{i}\right)}{\partial p_{H}}\right|_{\rho_{y} \text { const. }}<0, \text { and (b) }\left.\frac{\partial E\left(\pi_{i}\right)}{\partial \rho_{y}}\right|_{p_{H} \text { const. }} \sim-\varepsilon
$$

By (a), a uniform increase in the precision of the signals increases expected profits, as long as the correlation remains unchanged. In contrast to the case of Proposition 4.1

18 Part (a) implies Lemma la in Vives (1984), and (b) implies Lemma 3a. From (b), Proposition 1 in Fried (1984) follows. Parts (c) and (d) imply parts of Lemmas $1 \mathrm{~b}$ and $3 \mathrm{~b}$ in Vives (1984). The effects studied in Proposition 4.1 are nicely illustated in the Cournot model of Ponssard (1979), which also is a special case of our general model. 
(b), no relative information advantages of players are involved. Hence the precision of the private signal matters absolutely as well as in relation to the signals of the rival firms.

According to (b), an increase in the correlation of the signals - and hence in the correlation of equilibrium strategies - leaving the precision unchanged leads to higher expected profits for strategic complements and vice versa for strategic substitutes. Some intuition on this well-known result can be gained by considering a Cournot market with demand uncertainty (cf. Vives 1984): For a positive signal $y_{i}$, a higher correlation of signals implies a higher probability that the rival firms have received a high signal as well and supply a larger quantity. Since the reaction curves are downward-sloping, this induces a reduction of the own quantity $s_{i}$. As a result, $i$ reacts less sensitively to $y_{i}$, which reduces the expected profit.

The result also explains parts (c) and (d) of Proposition 4.1: an exogenous increase in the precision of an other player's signal (leaving the covariance unaffected) does not necessarily per se, i.e. because of an information advantage of the other firm, lead to a change of the expected profit, but rather by the increased correlation of strategies. ${ }^{19}$ Hence the profitability depends on the sign of $\varepsilon$.

\subsection{Complete pooling: correlation of strategies}

Setting $r_{i}=0 \forall i$ we obtain the case of complete information sharing: all $y_{i}$ are revealed without noise; all players have the same information. Noting that $r_{i} \bar{m}_{i}=0$ and $m_{i}=$ $m:=p_{H}-p_{N}$, we derive from Proposition 3.3

$$
\beta=c_{H} \frac{t_{H}+(n-1) p_{N} \frac{t_{H}-t_{N}}{p_{H}-p_{N}}}{2 \delta\left[p_{H}+(n-1) p_{N}\right]} \quad \gamma_{i}=\left(\beta-\frac{c_{H}}{\tilde{d}} \frac{t_{H}-t_{N}}{p_{H}-p_{N}}\right)\left(\frac{2 \delta}{d} \iota-\mathbf{e}_{\mathfrak{i}}\right) .
$$

Henceforth, we usually focus on the cases CV, PV, and PS introduced above. Consider the case of a common value (CV), where $t_{H}=t_{N}=t$. In essence, the State of Nature

19 Vives (1984) distinguishes the correlation effect and an information advantage of the rival firm, both affecting expected profits negatively. However, it does not follow from his analysis that there exists a negative information advantage effect if the correlation of the signals is held constant. 
is described by a real-valued random variable. Firms face identical cost and denand functions; all signals are equally reliable. Therefore, the strategies are affine in the sample mean of the signals, and all strategies are identical, as obtained from (4.5): $s_{i}=\alpha+(2 \delta \beta / \hat{d}) \iota^{\prime} \mathbf{y}$.

In the case of perfect signals (PS), we have $p_{H}=t_{H}$ and $p_{N}=t_{N}$. As can be seen in the expressions for $\beta$ and $\gamma_{i}$ in (4.5), all parameters of random variables cancel out: With complete exchange of information, all uncertainty vanishes; thus if $\tau$ is known to all players when the $s_{i}$ are set, the distribution of $\tau$ is irrelevant. ${ }^{20}$

Lemma 4.2 For the equilibrium strategies of an oligopoly with complete information sharing, we have for all $i, j(i \neq j)$

$$
\begin{aligned}
\operatorname{Var}\left(s_{i}^{C P}\right) & =\frac{c_{H}^{2}}{\hat{d}^{2}}\left[t_{H} \frac{T_{\oplus}}{P_{\oplus}}+(n-1) \theta^{\prime}\left(p_{N} \frac{T_{\oplus}}{P_{\oplus}}-t_{H}+\frac{4 \delta^{2}+(n-1) \varepsilon^{2}}{\tilde{d}^{2}}\left(t_{H}-t_{N}\right)\right)\right], \\
\operatorname{Cov}\left(s_{i}^{C P}, s_{j}^{C P}\right) & =\frac{c_{H}^{2}}{\hat{d}^{2}}\left[t_{H} \frac{T_{\oplus}}{P_{\oplus}}+(n-1) \theta^{\prime}\left(p_{N} \frac{T_{\oplus}}{P_{\oplus}}-t_{N}\right)-\frac{4 \delta^{2}+(n-1) \varepsilon^{2}}{\tilde{d}^{2}}\left(t_{H}-t_{N}\right) \theta^{\prime}\right]
\end{aligned}
$$

where $T_{\oplus}=t_{H}+(n-1) t_{N}$ is the variance of the mean of the $\tau_{i}$, i.e. of the mean deviance of demand or costs from the expected value (Shapiro 1986), $P_{\oplus}=p_{H}+(n-1) p_{N}$ and $\theta^{\prime}=\left(t_{H}-t_{N}\right) /\left(p_{H}-p_{N}\right)$.

Using Lemma 4.2 we obtain the sign of the correlation of the strategies as well as the direction of change with respect to the oligopoly without pooling.

Proposition 4.3 Let $\rho_{s}=\operatorname{Cov}\left(s_{i}, s_{j}\right) / \operatorname{Var}\left(s_{i}\right)$ denote the correlation of equilibrium strategies, where $\rho_{s}^{C P}$ and $\rho_{s}^{N P}$ refer to the complete-pooling and no-pooling cases, respectively. Then the signs of $\rho_{\mathrm{s}}$ and $\rho_{s}^{C P}-\rho_{3}^{N P}$ are given by the following table:

\begin{tabular}{|c|c|c|}
\hline & $\rho_{s}^{C P}$ & $\rho_{s}^{C P}-\rho_{s}^{N P}$ \\
\hline for $C V:$ & $=1$ & $>0$ \\
for $P V:$ & $\sim-\varepsilon$ & $\sim-\varepsilon$ \\
for $P S:$ & $\sim \frac{4 \delta^{2}+(n-1) \varepsilon^{2}}{\tilde{d}^{2}} t_{N}-\varepsilon \frac{\tilde{d}+\hat{d}_{d^{2}}}{d^{2}} t_{H}$ & $\sim-\varepsilon$ \\
\hline
\end{tabular}

${ }^{20}$ This observation is due to Shapiro (1986). 
In a common-value model information sharing always leads to an increase in the correlation of the strategies, whereas with private values or perfect signals, the correlation decreases for strategic substitutes and increases for strategic complements. Thus the conjecture that the correlation increases with a common value and decreases with private values (cf. Li 1985, Gal-Or 1986) is correct for Cournot oligopolies with substitute goods, but not in general. Note that with private values and strategic substitutes, information sharing leads to a negative correlation of previously uncorrelated strategies, whereas with perfect signals the sign of the correlation for complete pooling is ambiguous.

\subsection{Incentives to share I: contractual approach}

We now compare the expected profits without information sharing and with complete sharing which determine firms' incentives to enter into industry-wide contracts on information sharing. $\operatorname{Var}\left(s_{i}^{C P}\right)$ is given by Lemma 4.2 and $\operatorname{Var}\left(s_{i}^{N P}\right)$ by (4.2) and (4.4); therefore the profitability of information sharing is determined by

$$
\begin{aligned}
& E\left(\pi_{i}^{C P}\right)-E\left(\pi_{i}^{N P}\right)=\delta c_{H}^{2}\left\{\frac { 1 } { \hat { d } ^ { 2 } } \left[\left(t_{H}+(n-1) p_{N} \theta^{\prime}\right) \frac{T_{\oplus}}{P_{\oplus}}\right.\right. \\
& \left.\left.+(n-1) \theta^{\prime}\left(\frac{4 \delta^{2}+(n-1) \varepsilon^{2}}{\tilde{d}^{2}}\left(t_{H}-t_{N}\right)-t_{H}\right)\right]-\frac{p_{H} t_{H}^{2}}{\bar{d}^{2}}\right\}
\end{aligned}
$$

The sign of this difference does not depend on the sign of $c_{H}$. Hence at least for Cournot models, the source of uncertainty - demand or cost - affects the signs of the strategies but is irrelevant for expected profits. This has not been stated very clearly in previous papers, cf. Li (1985), Vives (1990).

Instead of the treating the PV and PS cases separately, we can derive more general results by taking an important similarity between these two cases into account: In

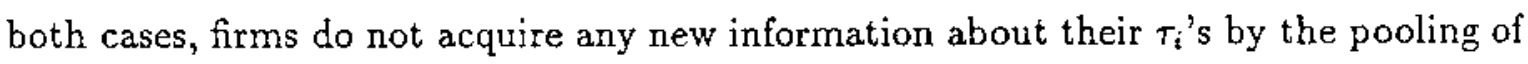
information. With perfect signals, firm i already knows $\tau_{i}$, whereas with uncorrelated signals, it cannot infer anything about $\tau_{i}$ from the other firms' signals. In the model, this is reflected in the fact that in both cases, $\hat{\mathbf{g}}_{\mathbf{i}}=0$ ).

Evaluation of (4.6) leads to 
Proposition 4.4 The difference of expected profits between complete pooling and no pooling is positive for $\hat{\mathrm{g}}_{\mathbf{i}}=0$, hence in particular for $P V$ and $P S$. For $C V$, the difference has the same sign as

$$
4 \delta(\delta-\varepsilon) p_{H}-(n-1) \varepsilon^{2}\left(p_{H}+n p_{N}\right)
$$

This expression is positive if $\mu:=\varepsilon / \delta$ is less than $2 /(n+1)$ and negative if $\mu$ is greater than $2(\sqrt{n}-1) /(n-1)<1$, and otherwise depends on the magnitudes of $p_{H}$ and $p_{N}$.

As corollaries follow the corresponding results of Clarke (1983), Fried (1984, Proposition 2), Li (1985, Proposition 2), Shapiro (1986, Theorem 1), Sakai (1986, Theorem 1), Kirby (1988, Proposition 2), and Vives (1984, Proposition 5).

As can be seen in the proof, the difference expressions for CV and PS contain the factors $p_{H}-p_{N}$ and $t_{H}-t_{N}$, respectively, reflecting the fact that with a common value and perfect signals firms know the State of Nature with certainty, so that the expected profits under CP and NP are equal. ${ }^{21}$

In a common-value Cournot oligopoly with homogeneous goods $(\varepsilon=\delta)$, complete sharing is unprofitable. For positive $\varepsilon$ information sharing is profitable if $\varepsilon$ is small - corresponding to a large degree of product differentiation, or, for quadratic costs, to quickly increasing marginal costs (cf. Kirby 1988) - and becomes unprofitable as $\varepsilon$ approaches $\delta$. Finally, for negative $\varepsilon$ (strategic complements) an agreement on information sharing is always profitable.

The most important consequence of Proposition 4.4 is that with perfect signals, complete pooling is always profitable, regardless of any other parameters of the model. This result is in sharp contrast with the interpretations of Fried (1984), Shapiro (1986), Li (1985), Sakai (1986) and Sakai/Yamato (1989), who have attributed the profitability of information sharing to the "private-value" character of their models or the uncertainty about costs as opposed to demand uncertainty. Rather, the result is completely determined by the assumption that firms have perfect knowledge of their own costs, or in general, of their $\tau_{i}$.

${ }^{21}$ Cf. Clarke's (1983) discussion of the Novshek/Sonnenschein (1982) model. 
Turning to private values, complete sharing is profitable if firms receive noisy signals, as long as the demand intercepts/costs are uncorrelated (Gal-Or 1986).

The unprofitability of information exchange in a homogeneous Cournot market with uncertainty about a common value is one of the earliest results of the literature. However, the proposition suggests that this is a rather exceptional case. Hence in general Clarke's (1983) argument that observing an agreement on information sharing may be taken as a prima-facie evidence for collusion does not apply.

\subsection{Incentives to share II: noncooperative approach}

Most authors of the information sharing literature have analyzed two-stage games in which firms simultaneously decide on their revelation behavior before playing the oligopoly game. The attractiveness of this approach grounds on information sharing (possibly) arising noncooperatively rather than by contract. However, taking a closer look at the economic situation reveals that binding commitments as to the revelation behavior of firms somehow have to be enforced anyway, since in general there is an ex-post incentive to deviate from the ex-ante decision. Thus the approach is not as noncooperative as it may seem at first glance.

Nevertheless, studying the two-stage game can yield important insights about the stability of information sharing arrangements. In particular, we will analyze under which circumstances firms have a dominant revelation strategy in the sense that they commit to a certain revelation behavior (e.g. always to reveal the own signal) regardless of how the other firms decide, in anticipation of the equilibrium of the oligopoly game resulting from the first-stage decisions.

In this subsection, we allow asymmetric revelation behavior, keeping in mind that non-revealing firms, too, have access to information revealed by other firms. However, because of the technical difficulties involved we exclude partial revelation; hence each firm has to decide whether to reveal completely or not at all. ${ }^{22}$

22 In analyzing the two-stage game, Vives (1984) and Gal-Or $(1985,1986)$ allow for partial revelation, whereas this is excluded by Li (1985) and implicitly by Fried (1984) and Sakai (1986). 
Without loss of generality we assume that the first $k$ players $(k \in\{0, \ldots, n\})$ reveal, whereas the last $n-k$ players conceal their information. A nonrevealing firm (for given $\mathrm{k}$ ) has an incentive to reveal if $E\left(\pi_{i}\left(s_{i}^{R, k+1}, \mathrm{~s}_{-\mathbf{i}}^{\mathbf{k}+1}\right)\right)-E\left(\pi_{i}\left(s_{i}^{N, k}, \mathrm{~s}_{-\mathbf{j}}^{\mathbf{k}}\right)\right)>0$, where $s_{i}^{R, k+1}$ denotes the strategy of a Revealing firm (increasing the number to $k+1$ ) and $s_{i}^{N, k}$ the strategy of a Nonrevealing firm (where the number of revealing firms remains $k$ ). If this inequality is valid for all $\mathrm{k}, \mathrm{i}$ has a dominant strategy to reveal (in the sense explained above), and vice versa if the inquality is never fulfilled (cf. Li 1985).

Setting $r_{i}=0$ for $i \in\{1, \ldots, k\}$ and $r_{i}=\infty$ for $i \in\{k+1, \ldots, n\}$ for a given $\mathrm{k}$, we can derive the equilibrium strategies for revealing and concealing firms from Proposition 3.3 , calculate their variances, and compute the expected profits. This leads to one of the main results of this section:

Proposition 4.5 For $C V$ and $\hat{\mathbf{g}_{\mathbf{i}}}=0$ (including $P V$ and $P S$ ), the sign of $E\left(\pi_{i}\left(s_{i}^{R, k+1}, \mathrm{~s}_{-\mathrm{i}}{ }^{k+1}\right)\right)-E\left(\pi_{i}\left(s_{i}^{N, k}, \mathrm{~s}_{-\mathrm{i}}{ }^{k}\right)\right)$ is independent of $k$. This difference is positive for $\hat{\mathrm{g}_{\mathrm{i}}}=0$ and $\sim-\varepsilon$ for $C V$, regardless of all other parameters.

There are many corresponding results in the literature: Proposition $3 \mathrm{in} \mathrm{Li}$ (1985) and Proposition 3 in Fried (1984) follow as corollaries; Gal-Or $(1985,1986)$ provides similar results for CV-and PV-duopolies and Vives (1984) for a CV-duopoly; Proposition 7 in Li (1985) qualitatively corresponds with the result for PS; Theorem 4 in Sakai (1986) states the corresponding result for PS-duopolies with arbitrary $\mathrm{D}$ and $\mathrm{T}$ matrices.

First of all, we observe that in all cases considered there are dominant revelation strategies. Furthermore, the result for PS supplements Proposition 4.4: the results obtained by Fried (1984), Li (1985), and Sakai (1986) have little to do with cost uncertainty or "private values" but are determined by the mere assumption of perfect signals.

Comparing Propositions 4.4 and 4.5, we see that for CV with strategic complements or with strategic substitutes and $\varepsilon$ close to $\delta$, and for PV and PS in any case, the equilibrium of the two-stage game is always efficient from the point of view of the firms. In contrast, for CV with strategic substitutes and small $\varepsilon$ (large degree of product 
differentiation) a prisoner's dilemma situation arises: complete sharing is profitable but does not occur in the two-stage game (cf. Vives 1984).

Although it might seem more reasonable to analyze exclusionary disclosure rules (i.e. where only revealing firms have access to information revealed by others; such rules have only been considered by Kirby [1988] and Shapiro [1986]), the results of this section suggest that this might not yield very interesting new insights, since in most of the considered cases the efficient solution already results from unilateral commitments on the revelation behavior, whereas "quid-pro-quo-agreements" (Kirby 1988) only become interesting in Prisoner's Dilemma situations where firms insist on the "quo". Of course, for exclusionary agreements among all $\mathrm{n}$ firms, the results of section 4.3 apply.

\subsection{Discussion of the results}

To explain the results of 4.3 and 4.4, we start with the common-value case (cf. Vives 1984). The pooling of information has two effects: first, each firm has better information about the State of Nature; second, strategies are perfecly correlated. The first effect increases expected profits, whereas the profitability of the second effect depends on the slope of the reaction curves (cf. Proposition 4.2). For strategic complements both effects are positive; thus information sharing is unambiguously profitable. For strategic substitutes (say, a Cournot market with substitute goods), the correlation effect is negative. According to Proposition 4.4, for $\varepsilon=\delta$ (homogeneous goods) the correlation effect outweighs the precision effect, implying that information sharing is not profitable. In contrast, a small $\varepsilon$ corresponds to a high degree of product differentiation. Here, the precision effect prevails since there is less intense competition, implying that the adverse effect of a higher correlation of strategies is smaller. Alternatively, if we have homogeneous goods and quadratic costs, information sharing is not advantageous as long as marginal costs rise slowly, whereas for quickly rising costs "errors" become expensive, hence it pays to acquire a better knowledge of the market (Kirby 1988).

If the $r_{i}$ 's are determined independently, the decision to reveal only depends on the 
correlation effect, since the knowledge of the State of Nature is not influenced by the own revelation behavior (cf. Proposition 3.2). This explains the difference between Propositions 4.4 and 4.5, which gives rise to a Prisoner's Dilemma.

We now turn to the PV and PS cases. Qualitatively, the results for these cases are very similar, which is not obvious as a PS model with highly correlated State-ofNature-components seems to have more in common with a common-value model than with a private-value model. As pointed out above, the essential similarity between PV and PS is that in both cases, the revealed signals improve the expectations about the information - and hence the strategies - of other players, but knowledge of the own partial market remains unchanged.

To understand the effect, it is helpful to distinguish two effects of the pooling of information as compared to the the situation without pooling: "direct adjustments", due to a better knowledge of the State of Nature, and "strategic adjustments", due to an improved knowledge of the rival firms' information and hence their actions. ${ }^{23}$, It then follows that in both cases PV and PS there are only strategic adjustments. From Proposition 4.5 we may thus conclude that for PV and PS, unilateral revelation of information to the other firms is profitable because and as long as this only induces strategic adjustments by the rival firms.

While strategic adjustments always alter the correlation of strategies in the direction profitable for the firms (Propositions $4.2 \mathrm{~b}, 4.3$, and 4.4), direct adjustments always lead to a higher correlation. Thus with strategic complements, both adjustments are profitable, whereas with strategic substitutes, the negative effect of highly correlated strategies may prevail.

Turning to intermediate cases between $\mathrm{PV}$ and $\mathrm{CV}$, in markets with strategic substitutes firms face a trade-off: a firm has an incentive to reveal her private information as long as this does not significantly improve other firms' knowledge of their $\tau_{j}$ which

23 This terminology is borrowed from Fried (1984), who uses the terms "direct adjustments" and "counteradjustments" in the same way, but in a slightly different context. Note that the separation of the two effects is only a conceptual one, because in general it is not possible to separate the equilibrium

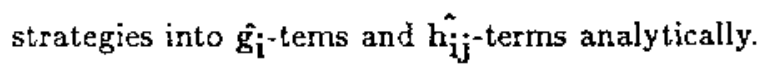


would induce direct adjustments by these firms and thereby lead to more intense competition (cf. Fried 1984, Proposition 4).

For a common value, we have the opposite case: by Proposition 3.3, the components of $\hat{\mathrm{g}}_{\mathfrak{i}}$ have their maximal value, implying maximal direct adjustments.

We close this dicussion with the observation that via the term $t_{N} p_{H}-t_{H} p_{N}$ the direct adjustments and hence the incentives to share information seem to be chiefly determined by the difference between $\rho_{\tau}$ and $\rho_{\eta}$, a conjecture we do not pursue further. ${ }^{24}$

Under the assumption of $c_{N}=0$, hence excluding Bertrand markets with cost uncertainty from the analysis, we have shown: For $\mathrm{CV}$ and strategic complements, and for PV and PS in any case, complete information pooling is an efficient equilibrium of the two-stage game, regardless of all other parameters. For CV and strategic substitutes, no pooling is the equilibrium solution. This solution is inefficient for a large degee of product differentiation and becomes efficient as goods become increasingly homogeneous. ${ }^{25}$ small $\varepsilon$ (in relation to $\delta$ ) $\varepsilon$ approaches $\delta$. Except for Gal-Or's (1986) Bertrand model with cost uncertainty, these statements summarize all results of the literature on the incentives to share information in symmetric models.

\subsection{Bertrand markets with cost uncertainty}

We briefly turn to Bertrand markets with cost uncertainty. As in this case $\pi_{i}^{A} \neq 0$, the analysis becomes considerably more complicated, and the results are much more ambiguous than in the other cases. Therefore, I will only summarize the results without presenting the formal analysis in full detail.

As to the method of analysis, it suffices to analyze $E\left(\pi_{i}^{A}\right)$ under different informational settings and then combine the results with the corresponding results for $\pi_{i}^{M}$

${ }^{24}$ At first glance, this contradicts Gal-Or's (1985) analysis of the role of correlated signal errors. But recall that in her model, $\rho_{\eta}$ is nonpositive, implying that in any case there is a significant difference between the correlations of $\tau$ and $\eta$.

${ }^{25}$ In in a Cournot model with quadratic costs (Kirby 1988), a large degree of product differentiation corresponds to a large coefficient of the quadratic term, i.e. quickly rising marginal costs. 
derived in 4.1-4.4. Referring to example (ii) in section 2, we assume for this subsection that $c_{H}=\delta$ and $c_{N}=\varepsilon$ (cf. Table 2 for the parameters of the Gal-Or [1986] model).

It turns out that in all three special cases (except for $\mathrm{CV}$ and small $\varepsilon$ ) complete pooling makes $\pi_{i}^{A}$ change in the opposite direction as $\pi_{i}^{M}$ (according to Proposition 4.4), leaving the direction of change of the total profit ambiguous.

In contrast to the simple results in the previous sections, the profitability of industrywide contracts on information sharing in general depends on the magnitudes of $\delta, \varepsilon$, and $n$. For example, for private values complete pooling is unprofitable if $\varepsilon>0$ (complements) or $n \leq 3$, but profitable if $\varepsilon \rightarrow-\frac{1}{\pi-1} \delta$ (homogeneous goods).

Proceeding in the same way as in 4.4, we can evaluate $\pi^{A}$ for a nonrevealing and a revealing firm, respectively, when there are $\mathrm{k}$ other revealing firms, and add the resulting differences in profits to our previous results. Similarly as above, the profitabilty of unilateral information revelation depends on the magnitudes of $\delta, \varepsilon$, and $n$. Moreover, in general there do not even exist dominant revelation strategies.

Only in the case of private values there is a dominant revelation strategy. This strategy depends on the difference between expected profits for unilateral revelation vs. concealing, which has the same sign as $-4(2 \delta-\varepsilon)-(n-1) \varepsilon(4 \delta-3 \varepsilon)$. If $\varepsilon>0$ or $n=2$, to conceal information is a dominant revelation strategy. The latter result corresponds to the case of a duopoly considered by Gal-Or (1986). However, for negative $\varepsilon$ and $n \rightarrow \infty$, revealing becomes a dominant strategy. Thus in contrast to Cournot markets or to Bertrand markets with demand uncertainty, the incentives to share information depend on the number of firms; results obtained for duopolies do not extend to larger markets.

Comparing the results for the contractual and the noncooperative approach, we obtain that for $n=2$ and $n \rightarrow \infty$ the equilibrium outcome of the two-stage game is efficient, whereas there is range of values for $\mathrm{n}$ (or $\varepsilon$ ) where complete sharing is efficient, but does not occur. 


\section{Concluding Remarks}

The model analyzed in this paper is a generalization of most models in the literature on information sharing in oligopoly. The n-firm oligopoly model allows for prices or quantities as strategic variables, product differentiation, substitutes or complements, uncertainty about the demand intercept of the respective partial market or uncertainty about the own marginal cost, arbitrarily correlated dernand intercepts/costs, noisy or perfect private signals with asymmetric precisions and correlated signal errors, and asymmetric information revelation behavior with complete or partial revelation.

In a two-stage game, firms first decide on their revelation behavior, then receive private information about the State of Nature and reveal it according to their prior decision, and finally set prices or quantities noncooperatively.

Using the general framework to compare the assumptions and results of previous models, it is argued that the distinction of "common-value" and "private-value" models prevailing in the literature is inadequate. More naturally, a common value emerges as the limit case of increasingly correlated demand intercepts/costs. We introduce a new category for models in which firms have perfect knowledge of their own costs/demand.

The analysis of the incentives to share information reveals that for Cournot markets and for Bertrand markets with demand uncertainty, there are some simple principles underlying almost all previous results: With perfect signals or uncorrelated demands/costs, or with a common value and strategic complements, complete information pooling is an efficient equilibrium of the two-stage game, regardless of all other parameters. With a common value and strategic substitutes, no pooling is the equilibrium solution. This solution is efficient in Cournot markets with homogeneous goods and inefficient for a large degree of product differentiation. It is uncovered that the profitability of information sharing in most models with cost uncertainty is determined by the mere assumption that firms know their own costs with certainty, which refutes previous interpretations attributing these results to other factors. For an exceptional case, Bertrand markets with cost uncertainty, the incentives are rather ambiguous. It turns out that results derived for duopoly models (Gal-Or 1986) may be reversed in 
the case of many firms. We suggest a new explanation for the incentives to reveal private information which leads to an improved understanding of the role of Cournot vs. Bertrand markets, demand vs. cost uncertainty, and the slopes of the reaction curves: (i) Letting the rivals acquire a better knowledge of their respective partial markets leads to a higher correlation of strategies, the profitability of which is determined by the slope of the reaction curves. (ii) Letting the rivals acquire a better knowledge of one's own information (and hence action) is always profitable. The incentive to reveal information is then determined by the sum of these two effects.

An analysis of the welfare effects of information sharing (excluding Bertrand markets with cost uncertainty), not included in this paper, leads to less clear-cut results: The intuitive conjecture, often found in the nonformal literature, that without collusion information sharing is socially beneficial fails to take into account the impact of a change in the correlation of strategies on profits and the effect on consumers' surplus. In general, producers and consumers have conflicting interests, making a weighting of these interests necessary (cf. Shapiro 1986). Only in rare cases do profits and consumers' surplus both increase due to information exchange.

In many cases, the direction of change of consumer surplus and total welfare depends on the magnitudes of the parameters of the demand system and the number of firms. Nevertheless, for both Cournot (cost or demand uncertainty) and Bertrand (demand uncertainty) the equilibrium behavior of firms in most cases is socially optimal (in terms of overall welfare) as well. Complications arise, however, if costs of information sharing (verification costs) lead to information exchange still being profitable for the firms but imposing a welfare loss for society (Shapiro 1986).

If one is willing to draw policy conclusions from the model developed here, then antitrust authorities should prevent observed information exchange only if (i) this is likely to lead to collusion or (ii) if a high weight is placed on consumers' surplus or (iii) if costs of information exchange are large.

The understanding of the incentives to share information could be further enhanced by analyzing situations where firms have differently precise private information. Clarke 
(1983), Fried (1984), and Sakai (1986) have made contributions on this topic, and the framework of Sections 2 and 3 allows for a treatment on a more general level. Moreover, the analysis of the oligopoly game provides a basis for analyzing the incentives to acquire private information, along the lines of $\mathrm{Li}, \mathrm{McKelvey,} \mathrm{and} \mathrm{Page} \mathrm{(1987).} \mathrm{All} \mathrm{this} \mathrm{is} \mathrm{left}$ for future research. 


\section{A Proofs of Lemmas and Propositions}

Throughout the appendix, I will denote the n-dimensional unit matrix, and $\iota:=$ $(1, \ldots, 1)^{\prime}$. Moreover, let $\overline{\mathrm{I}}:=\iota \iota^{\prime}-\mathbf{I}$. Additional symbols will be defined in several proofs and used subsequently. Appendix B provides a list of the symbol definitions.

\section{Proof of Proposition 3.2:}

1. We start with a matrix-algebraic result which will be used in the proofs of Section 3. For matrices $\mathbf{A}(p \times p$, nonsingular), $\mathbf{U}, \mathbf{V}(q \times p)$, and $\mathbf{S}(q \times q)$ we have

$$
\left(\mathbf{A}+\mathrm{U}^{\prime} \mathbf{S V}\right)^{-1}=\mathbf{A}^{-1}-\mathbf{A}^{-1} \mathbf{U}^{\prime} \mathbf{S}\left(\mathbf{S}+\mathbf{S V A}^{-1} \mathbf{U}^{\prime} \mathbf{S}\right)^{-1} \mathrm{SVA}^{-1}
$$

(see Madansky 1976, p. 9) Now let $\mathbf{a} \in \mathbb{R}^{n}, b \in \mathbb{R}$ and $\mathbf{M}=\operatorname{diag}(\mathbf{a})+b \iota^{\prime}$. Then from (A.1) it follows that

$$
\mathbf{M}^{-1}=\operatorname{diag}(\overline{\mathbf{a}})-\frac{b}{1+b \iota^{\prime} \mathbf{\overline { a }}} \overline{\mathbf{a}} \bar{\prime}^{\prime}, \quad \text { where } \overline{\mathbf{a}}=\left(\frac{1}{a_{1}}, \ldots, \frac{1}{a_{n}}\right)^{\prime} .
$$

In particular, if $\mathbf{M}=a \mathbf{I}+b \iota \iota^{\prime}(a, b \in \mathbb{R})$, then

$$
\mathbf{M}^{-1}=\frac{1}{a} \mathbf{I}-\frac{b}{a(a+b n)} \iota \iota^{\prime}
$$

2. Since $\tau, \boldsymbol{\eta}$, and $\boldsymbol{\xi}$ are independent, we have

$$
\operatorname{Cov}\left(\begin{array}{c}
\tau \\
\mathbf{y} \\
\hat{\mathbf{y}}
\end{array}\right)=\left(\begin{array}{ccc}
\mathbf{T} & \mathbf{T} & \mathbf{T} \\
\mathbf{T} & \mathbf{P} & \mathbf{P} \\
\mathbf{T} & \mathbf{P} & \mathbf{Q}
\end{array}\right)
$$

Writing $\mathbf{T}$ and $\mathbf{P}$ as rows of column vectors, $\mathbf{T}=\left(\mathbf{t}_{\mathbf{1}}, \ldots, \mathbf{t}_{\mathfrak{n}}\right)$ and $\mathbf{P}=\left(\mathbf{p}_{1}, \ldots, \mathbf{p}_{\mathbf{n}}\right)$, we obtain

$$
\operatorname{Cov}\left(\begin{array}{c}
\tau_{i} \\
y_{i} \\
\hat{\mathbf{y}}
\end{array}\right)=\left(\begin{array}{ccc}
t_{H} & t_{H} & \mathbf{t}_{\mathbf{i}}^{\prime} \\
t_{H} & p_{i \mathbf{i}} & \mathbf{p}_{\mathbf{i}}^{\prime} \\
\mathbf{t}_{\mathbf{i}} & \mathbf{P}_{\mathbf{i}} & \mathbf{Q}
\end{array}\right) \text { and } \operatorname{Cov}\left(\begin{array}{c}
y_{j} \\
y_{i} \\
\hat{\mathbf{y}}
\end{array}\right)=\left(\begin{array}{ccc}
p_{j i} & p_{N} & \mathbf{p}_{\mathbf{j}}^{\prime} \\
p_{N} & p_{i i} & \mathbf{p}_{\mathbf{i}}^{\prime} \\
\mathbf{p}_{\mathbf{j}} & p_{\mathbf{i}} & \mathbf{Q}
\end{array}\right) \text {. }
$$

Therefore (Cf. Schönfeld 1969, p. 98)

$$
\begin{aligned}
& E\left(\tau_{i} !\left(\begin{array}{c}
y_{i} \\
\hat{\mathbf{y}}
\end{array}\right)\right)=\left(t_{H}, \mathbf{t}_{\mathrm{i}}{ }^{\prime}\right)\left(\begin{array}{cc}
p_{i i} & \mathbf{p}_{\mathbf{i}}^{\prime} \\
\mathbf{p}_{\mathrm{i}} & \mathbf{Q}
\end{array}\right)^{-1}\left(\begin{array}{c}
y_{i} \\
\hat{\mathbf{y}} .
\end{array}\right) \text { and } \\
& E\left(y_{j} \mid\left(\begin{array}{c}
y_{i} \\
\hat{\mathrm{y}}
\end{array}\right)\right)=\left(p_{N} \mathbf{p}_{\mathrm{j}}^{\prime}\right)\left(\begin{array}{cc}
p_{i i} & \mathbf{p}_{\mathrm{i}}^{\prime} \\
\mathbf{p}_{\mathrm{i}} & \mathbf{Q}
\end{array}\right)^{-1}\left(\begin{array}{c}
y_{i} \\
\hat{\mathbf{y}}
\end{array}\right) .
\end{aligned}
$$


Evaluating the partitioned matrix in the equations above (cf. Theil 1971, p.17-18) yields

$$
\begin{aligned}
& \left(\begin{array}{ll}
p_{i j} & \mathrm{P}_{\mathrm{i}}^{\prime} \\
\mathrm{P}_{\mathrm{i}} & \mathrm{Q}
\end{array}\right)^{-1}=\left(\begin{array}{ll}
C_{1} & \mathrm{C}_{2} \\
\mathrm{C}_{3} & \mathrm{C}_{4}
\end{array}\right), \quad \text { where } \\
& C_{1}=\left(p_{i i}-\mathbf{p}_{\mathbf{i}}^{\prime} \mathbf{Q}^{-1} \mathbf{p}_{\mathbf{i}}\right)^{-1} \\
& \mathrm{C}_{2}=-C_{1} \mathrm{P}_{\mathrm{i}}^{\prime} \mathrm{Q}^{-1} \\
& \mathrm{C}_{3}=-C_{1} \mathrm{Q}^{-1} \mathrm{Pi} \\
& \mathbf{C}_{4}=\mathbf{Q}^{-1}+C_{1} \mathbf{Q}^{-1} \mathbf{p}_{\mathrm{i}} \mathbf{p}_{i}^{\prime} \mathbf{Q}^{-1} \quad(n \times n)
\end{aligned}
$$

Thus we have

$$
\begin{aligned}
& E\left(\tau_{i} \mid\left(\begin{array}{c}
y_{i} \\
\hat{\mathbf{y}}
\end{array}\right)\right)=g_{i} y_{i}+\dot{\mathbf{g}}_{\mathrm{i}}^{\prime} \hat{\mathbf{y}} \text { and } E\left(y_{j} \mid\left(\begin{array}{c}
y_{i} \\
\hat{\mathbf{y}}
\end{array}\right)\right)=h_{i j} y_{i}+\hat{\mathrm{h}}_{\mathrm{ij}}^{\prime} \hat{\mathbf{y}} \text {, where } \\
& g_{i}=t_{H} C_{1}+\mathbf{t}_{\mathbf{i}}^{\prime} \mathbf{C}_{3}=\frac{t_{H}-\mathbf{t}_{\mathbf{i}}^{\prime} \mathbf{Q}^{-1} \mathbf{p}_{\mathbf{i}}}{p_{i i}-\mathbf{p}_{\mathbf{i}}^{\prime} \mathbf{Q}^{-1} \mathbf{p}_{\mathbf{i}}}, \\
& \hat{\mathbf{g}}_{\mathbf{i}}^{\prime}=t_{H} \mathrm{C}_{2}+\mathrm{t}_{\mathrm{i}}^{\prime} \mathrm{C}_{\mathbf{4}}=\left(\mathrm{t}_{\mathrm{i}}^{\prime}-g_{\mathrm{i}} \mathrm{p}_{\mathrm{i}}^{\prime}\right) \mathrm{Q}^{-1} \text { or } \hat{\mathrm{g}}_{\mathbf{i}}=\mathrm{Q}^{-1}\left(\mathrm{t}_{\mathrm{i}}-g_{\mathrm{i}} \mathrm{p}_{\mathrm{i}}\right) \text {, } \\
& h_{i j}=p_{N} C_{1}+\mathrm{p}_{\mathrm{j}}^{\prime} \mathrm{C}_{3}=\frac{p_{N}-\mathrm{p}_{\mathrm{j}}^{\prime} \mathrm{Q}^{-1} \mathrm{p}_{\mathrm{i}}}{p_{i i}-\mathrm{p}_{\mathrm{i}}^{\prime} \mathrm{Q}^{-1} \mathrm{p}_{\mathrm{j}}} \text {, } \\
& \hat{\mathrm{h}}_{\mathrm{ij}}^{\prime}=p_{N} \mathbf{C}_{2}+\mathbf{p}_{\mathbf{j}}^{\prime} \mathbf{C}_{\mathbf{4}}=\left(\mathrm{p}_{\mathbf{j}}^{\prime}-h_{i j} \mathbf{p}_{\mathbf{i}}^{\prime}\right) \mathbf{Q}^{-1} \text { or } \hat{\mathrm{h}}_{\mathrm{ij}}=\mathrm{Q}^{-1}\left(\mathrm{p}_{\mathbf{j}}-h_{\mathrm{ij}} \mathrm{p}_{\mathrm{i}}\right) \text {. }
\end{aligned}
$$

3. For $m_{i}:=p_{i i}-p_{N}+r_{i}, \mathbf{m}=\left(m_{1}, \ldots, m_{n}\right)^{\prime}, \overline{\mathbf{m}}:=\left(1 / m_{1}, \ldots, 1 / m_{n}\right)^{\prime}$ and $M:=\iota^{\prime} \overline{\mathbf{m}}$ it follows that $\mathbf{Q}=\operatorname{diag}(\mathbf{m})+p_{N} \iota \iota^{\prime}$ and by $(\mathrm{A} .2) \mathbf{Q}^{-1}=\operatorname{diag}(\overline{\mathbf{m}})-p_{N} \overline{\mathbf{m}} \overline{\mathbf{m}}^{\prime} /\left(1+p_{N} M\right)$. Writing $\mathrm{p}_{\mathrm{i}}=p_{N} \iota+\left(p_{i \mathrm{i}}-p_{N}\right) \mathbf{e}_{\mathbf{i}}$ we get

$$
\begin{aligned}
\mathbf{Q}^{-1} \mathbf{p i}= & {\left[\operatorname{diag}(\overline{\mathbf{m}})-\frac{p_{N}}{1+p_{N} M} \overline{\mathbf{m}}^{\overline{\mathbf{m}}^{\prime}}\right]\left[p_{N} \iota+\left(p_{i \mathrm{i}}-p_{N}\right) \mathrm{e}_{\mathrm{i}}\right] } \\
= & p_{N} \overline{\mathbf{m}}+\left(p_{i \mathrm{i}}-p_{N}\right) \bar{m}_{i} \mathrm{e}_{\mathbf{i}}-\frac{p_{N}^{2} M}{1+p_{N} M} \overline{\mathbf{m}}-\frac{p_{N}\left(p_{i \mathrm{i}}-p_{N}\right)}{1+p_{N} M} \bar{m}_{\mathbf{i}} \overline{\mathbf{m}} \\
= & \frac{1}{1+p_{N} M}\left\{\left[p_{N}\left(1+p_{N} M\right)-p_{N}^{2} M-p_{N}\left(p_{i i}-p_{N}\right) \bar{m}_{i}\right] \overline{\mathbf{m}}\right. \\
& \left.+\left(p_{i i}-p_{N}\right)\left(1+p_{N} M\right) \bar{m}_{i} \mathbf{e}_{\mathrm{i}}\right\} \\
= & \frac{1}{1+p_{N} M}\left\{p_{N}\left[1-\bar{m}_{i}\left(p_{i i}-p_{N}\right)\right] \overline{\mathbf{m}}+\bar{m}_{i}\left(p_{i i}-p_{N}\right)\left(1+p_{N} M\right) \mathbf{e}_{\mathbf{i}}\right\} .
\end{aligned}
$$

Let $\mathbf{w}:=w_{N} \iota+\left(w_{H}-w_{N}\right) \mathbf{e}_{\mathbf{j}}$ for arbitrary $w_{H}, w_{N}$; and let $\delta_{i j}$ denote the Kroneckerdelta. Then

$$
\left(1+p_{N} M\right) \mathbf{w}^{\prime} \mathbf{Q}^{-1} \mathbf{p}_{\mathbf{i}}
$$




$$
\begin{aligned}
= & {\left[w_{N} \iota^{\prime}+\left(w_{H}-w_{N}\right) \mathbf{e}_{\mathbf{j}}^{\prime}\right\}\left[p_{N}\left(1-\bar{m}_{i}\left(p_{i i}-p_{N}\right)\right) \overline{\mathbf{m}}+\bar{m}_{i}\left(p_{i i}-p_{N}\right)\left(1+p_{N} M\right) \mathbf{e}_{\mathbf{i}}\right] } \\
= & w_{N} p_{N}\left(1-\bar{m}_{i}\left(p_{i i}-p_{N}\right)\right) M+w_{N} \bar{m}_{i}\left(p_{i i}-p_{N}\right)\left(1+p_{N} M\right)+ \\
& p_{N}\left(w_{H}-w_{N}\right)\left(1-\bar{m}_{i}\left(p_{i i}-p_{N}\right)\right) \bar{m}_{j}+\bar{m}_{\mathbf{i}}\left(w_{H}-w_{N}\right)\left(p_{i i}-p_{N}\right)\left(1+p_{N} M\right) \delta_{i j}
\end{aligned}
$$

4. For $i=j$ this expression reduces to

$$
\left(1+p_{N} M\right) \mathbf{w}^{\prime} \mathbf{Q}^{-1} \mathrm{p}_{\mathrm{i}}=p_{N}\left[\left(w_{H}-w_{N}\right)\left(p_{i i}-p_{N}\right) \bar{m}_{i}+w_{N}\right]\left(M-\bar{m}_{i}\right)+w_{H} p_{i i} \bar{m}_{i}
$$

and after some steps we obtain

$$
\left(1+p_{N} M\right)\left(w_{H}-\mathbf{w}^{\prime} \mathbf{Q}^{-1} \mathbf{p}_{\mathbf{i}}\right)=\left[w_{H}+p_{N}\left(w_{H}-w_{N}\right)\left(M-\bar{m}_{i}\right)\right] r_{i} \bar{m}_{i} .
$$

Substituting $\mathbf{P}_{\mathbf{i}}=p_{N} \iota+\left(p_{i i}-p_{N}\right) \mathbf{e}_{\mathbf{j}}$ and $\mathbf{t}_{\mathbf{i}}=t_{N} \iota+\left(t_{H}-t_{N}\right) \mathbf{e}_{\mathbf{i}}$ for $\mathbf{w}$ yields

$$
g_{i}=\frac{1+p_{N} M}{1+p_{N} M} \quad \frac{t_{H}-t_{\mathbf{i}}^{\prime} \mathbf{Q}^{-1} \mathbf{P}_{\mathbf{i}}}{p_{i \mathrm{i}}-\mathbf{P}_{\mathbf{i}}^{\prime} \mathbf{Q}^{-1} \mathbf{P}_{\mathbf{i}}}=\frac{t_{H}+p_{N}\left(t_{H}-t_{N}\right)\left(M-\bar{m}_{i}\right)}{p_{i i}+p_{N}\left(p_{i i}-p_{N}\right)\left(M-\bar{m}_{i}\right)}=\frac{\theta_{i}}{D_{i}}
$$

where $\theta_{i}:=t_{H}+p_{N}\left(t_{H}-t_{N}\right)\left(M-\bar{m}_{i}\right)$ and $D_{i}:=p_{i i}+p_{N}\left(p_{i i}-p_{N}\right)\left(M-\bar{m}_{i}\right)$.

5. Since $\mathrm{t}_{\mathrm{i}}-g_{\mathrm{i}} \mathrm{p}_{\mathrm{i}}=\left(t_{N}-g_{i} p_{N}\right) \iota+\left[\left(t_{H}-t_{N}\right)-g_{i}\left(p_{i i}-p_{N}\right)\right] \mathrm{e}_{\mathrm{i}}$, we have

$$
\begin{aligned}
\hat{\mathbf{g}}_{\mathbf{i}}= & \mathbf{Q}^{-1}\left(t_{\mathbf{i}}-g_{i} \mathrm{p}_{\mathbf{i}}\right) \\
= & {\left[\operatorname{diag}(\overline{\mathbf{m}})-\frac{p_{N}}{1+p_{N} M} \overline{\mathbf{m}} \overline{\mathbf{m}}^{\prime}\right]\left[\left(t_{N}-g_{i} p_{N}\right) \iota+\left(\left(t_{H}-t_{N}\right)-g_{i}\left(p_{i i}-p_{N}\right)\right) \mathbf{e}_{\mathbf{i}}\right] } \\
= & \left(t_{H}-t_{N}-g_{i}\left(p_{i i}-p_{N}\right)\right) \bar{m}_{\mathbf{i}} \mathbf{e}_{\mathbf{i}} \\
& +\frac{1}{1+p_{N} M}\left[t_{N}-g_{i} p_{N}-p_{N}\left(t_{H}-t_{N}-g_{i}\left(p_{i i}-p_{N}\right)\right) \bar{m}_{i}\right] \overline{\mathbf{m}}
\end{aligned}
$$

Making use of (A.7) we obtain

$$
\begin{aligned}
& t_{H}-t_{N}-g_{i}\left(p_{i i}-p_{N}\right)=\frac{1}{D_{i}}\left(t_{H} p_{N}-t_{N} p_{i j}\right) \quad \text { and } \\
& t_{N}-g_{i} p_{N}=\frac{1}{D_{i}}\left(1+p_{N}\left(M-\bar{m}_{i}\right)\right)\left(t_{N} p_{i i}-t_{H} p_{N}\right), \quad \text { so that } \\
& t_{N}-g_{i} p_{N}-p_{N}\left(t_{H}-t_{N}-g_{i}\left(p_{i i}-p_{N}\right)\right) \bar{m}_{i}=\frac{1}{D_{i}}\left(1+p_{N} M\right)\left(t_{N} p_{i i}-t_{H} p_{N}\right)
\end{aligned}
$$

and finally $\hat{\mathbf{g}}_{\mathbf{i}}=\left(1 / D_{i}\right)\left(t_{N} p_{i i}-t_{H} p_{N}\right)\left(\overline{\mathbf{m}}-\bar{m}_{i} \mathbf{e}_{\mathbf{i}}\right)$.

6. We now compute $h_{i j}=\left(p_{N}-\mathbf{p}_{\mathbf{j}}^{\prime} \mathbf{Q}^{-1} \mathbf{p}_{\mathbf{i}}\right) /\left(p_{i i}-\mathbf{p}_{\mathbf{i}}^{\prime} \mathbf{Q}^{-1} \mathbf{p}_{\mathbf{i}}\right)$. Substituting $\mathbf{p}_{\mathrm{j}}=p_{N} \iota+$ $\left(p_{j j}-p_{N}\right) e_{\mathbf{j}}$ for $w$ in (A.5) we get

$$
\left(1+p_{N} M\right) \mathbf{P}_{\mathbf{j}}^{\prime} \mathbf{Q}^{-1} \mathbf{p}_{\mathbf{i}}
$$


$=p_{N}^{2}\left(1-\vec{m}_{i}\left(p_{i i}-p_{N}\right)\right) M+p_{N}\left(p_{i i}-p_{N}\right)\left(1+p_{N} M\right) \bar{m}_{i}$

$$
+p_{N}\left(p_{j j}-p_{N}\right)\left(1-\bar{m}_{i}\left(p_{i i}-p_{N}\right)\right) \bar{m}_{j}
$$

$=p_{N}\left[p_{N} M+\left(p_{i i}-p_{N}\right) \bar{m}_{i}+\left(p_{j j}-p_{N}\right) \bar{m}_{j}-\left(p_{i i}-p_{N}\right)\left(p_{j j}-p_{N}\right) \bar{m}_{i} \bar{m}_{j}\right]$, hence

$\left(1+p_{N} M\right)\left(p_{N}-\mathbf{p}_{\mathbf{j}}^{\prime} \mathbf{Q}^{-1} \mathbf{p}_{\mathbf{i}}\right)=p_{N}\left[1-\left(p_{i i}-p_{N}\right) \bar{m}_{\mathbf{i}}\right]\left[1-\left(p_{j j}-p_{N}\right) \bar{m}_{j}\right]=p_{N} r_{j} r_{j} \bar{m}_{i} \bar{m}_{j}$

According to (A.6), $D_{i} r_{i} \bar{m}_{i}=\left(1+p_{N} M\right)\left(p_{i j}-\mathbf{p}_{\mathbf{i}}^{\prime} \mathbf{Q}^{-1} \mathbf{p}_{\mathbf{i}}\right)$, implying that $h_{i j}=p_{N} r_{j} \bar{m}_{j} / D_{i}$.

7. Finally we evaluate $\hat{h}_{\mathrm{jj}}=\mathrm{Q}^{-1}\left(\mathrm{P}_{\mathbf{j}}-h_{i j} \mathrm{p}_{\mathrm{i}}\right)$. Since

$$
\begin{aligned}
& \mathbf{p}_{\mathbf{j}}-h_{i j} \mathbf{p}_{\mathbf{i}}=p_{N}\left(1-h_{i j}\right) \iota+\left(p_{j j}-p_{N}\right) \mathbf{e}_{\mathbf{j}}-h_{i j}\left(p_{i j}-p_{N}\right) \mathbf{e}_{\mathbf{i}}, \quad \text { we get } \\
\hat{h}_{\mathrm{ij}}= & {\left[\operatorname{diag}(\overline{\mathbf{m}})-\frac{p_{N}}{1+p_{N} M} \overline{\mathbf{m}} \overline{\mathbf{m}}^{\prime}\right]\left[p_{N}\left(1-h_{i j}\right) \iota+\left(p_{j j}-p_{N}\right) \mathbf{e}_{\mathbf{j}}-h_{i j}\left(p_{i i}-p_{N}\right) \mathbf{e}_{\mathbf{i}}\right] } \\
= & \frac{1}{1+p_{N} M}\left[p_{N}\left(1-h_{i j}\right)\left(1+p_{N} M\right)-p_{N}^{2}\left(1-h_{i j}\right) M-p_{N}\left(p_{j j}-p_{N}\right) \bar{m}_{j}\right. \\
& \left.-h_{i j}\left(p_{i i}-p_{N}\right)\left(1+p_{N} M\right)+h_{i j} p_{N}\left(p_{i i}-p_{N}\right) \bar{m}_{\mathbf{i}}\right] \overline{\mathbf{m}} \\
& +\left(p_{j j}-p_{N}\right) \bar{m}_{j} \mathbf{e}_{j}+h_{i j}\left(p_{i i}-p_{N}\right)\left(\overline{\mathbf{m}}-\bar{m}_{i} \mathbf{e}_{\mathbf{i}}\right)
\end{aligned}
$$

Using the definitions of $h_{i j}, D_{i}$, and $\bar{m}_{j}$ it can be shown that the expression in square brackets in the last line of (A.8) vanishes, yielding the expression in the proposition.

\section{Proof of Proposition 3.3:}

In addition to the symbols introduced in the proposition, define:

$$
\begin{aligned}
& \bar{v}_{i}=1 / v_{i} \quad \overline{\mathrm{v}}=\left(\bar{v}_{1}, \ldots, \bar{v}_{n}\right)^{\prime} \quad \bar{m}_{i}=r_{i} \bar{m}_{i} \\
& \overline{\mathrm{m}}=\left(\tilde{m}_{1}, \ldots, \tilde{m}_{n}\right)^{\prime} w_{i}=\theta_{i} / v_{i} \quad \mathbf{w}=\left(w_{1}, \ldots, w_{n}\right)^{\prime} \\
& \omega=\tilde{\mathbf{m}}^{\prime} \overline{\mathrm{v}} \quad \chi=1+\varepsilon p_{N} \omega \quad \psi=\tilde{\mathbf{m}}^{\prime} \mathbf{w}
\end{aligned}
$$

1. Identification of the unknown coefficients with the corresponding terms in (3.2) leads to the following system of equations in $\alpha_{i}, \beta_{i}, \gamma_{i}$ for all $i=1, \ldots, n$ :

$$
\begin{aligned}
2 \delta \alpha_{i} & =b_{i i}-\varepsilon \sum_{j \neq i} \alpha_{j} \\
2 \delta \beta_{i} & =c_{H} g_{i}-\varepsilon \sum_{j \neq i} \beta_{j} h_{i j} \\
2 \delta \gamma_{i} & =c_{H} \hat{\mathbf{g}}_{\mathbf{i}}-\varepsilon \sum_{j \neq i}\left(\beta_{j} \hat{\mathrm{h}}_{\mathbf{i j}}+\boldsymbol{\gamma}_{j}\right) .
\end{aligned}
$$


2. We first compute the $\alpha_{i}$. Equation (A.9) implies

$$
2 \delta \alpha_{i}+\varepsilon \sum_{j \neq i} \alpha_{j}=b_{i i} \quad \forall i \quad \text { or } \quad \alpha=\tilde{\mathbf{D}}^{-1} \overline{\mathrm{b}}
$$

where $\tilde{\mathbf{D}}:=\tilde{d} \mathbf{I}+\varepsilon \overline{\mathbf{I}}$ and $\tilde{\mathrm{b}}=\left(b_{11}, \ldots, b_{n n}\right)^{\prime}$. Then by (A.3),

$$
\alpha=\frac{1}{\tilde{d}}\left(\tilde{\mathrm{b}}-\frac{\varepsilon}{\hat{d}}\left(\iota^{\prime} \tilde{\mathrm{b}}\right) \iota\right) \quad \text { and } \quad \alpha_{i}=\frac{1}{\tilde{d}}\left(b_{i i}-\frac{\varepsilon}{\hat{d}} \iota^{\prime} b_{H}\right)
$$

3. Equation (A.10) implies

$$
\left(\begin{array}{cccc}
2 \delta & \varepsilon h_{12} & \cdots & \varepsilon h_{1 n} \\
\varepsilon h_{21} & 2 \delta & & \vdots \\
\vdots & & \ddots & \vdots \\
\varepsilon h_{n 1} & \cdots & \cdots & 2 \delta
\end{array}\right)\left(\begin{array}{c}
\beta_{1} \\
\vdots \\
\beta_{n}
\end{array}\right)=c_{H}\left(\begin{array}{c}
g_{1} \\
\vdots \\
g_{n}
\end{array}\right)
$$

Using $g_{i}=\theta_{i} / D_{i}$, the right-hand side of (A.12) equals

$$
c_{H}\left(\frac{\theta_{1}}{D_{1}}, \ldots, \frac{\theta_{n}}{D_{n}}\right)^{\prime}=c_{H} \operatorname{diag}\left(\frac{1}{D_{1}}, \ldots, \frac{1}{D_{n}}\right)^{\prime} \theta=: \mathbf{b}^{*} .
$$

Furthermore, due to Proposition 3.2 we have

$$
\left(\begin{array}{ccc}
h_{11} & \cdots & h_{1 n} \\
\vdots & \ddots & \vdots \\
h_{n 1} & \cdots & h_{n n}
\end{array}\right)=p_{N}\left(\begin{array}{c}
1 / D_{1} \\
\vdots \\
1 / D_{n}
\end{array}\right)\left(r_{1} \bar{m}_{1}, \ldots, r_{n} \bar{m}_{n}\right)
$$

Now define

$$
\begin{aligned}
\mathrm{D}^{*} & :=\varepsilon p_{N}\left(\begin{array}{c}
1 / D_{1} \\
\vdots \\
1 / D_{n}
\end{array}\right)\left(r_{1} \bar{m}_{1}, \ldots, r_{n} \bar{m}_{n}\right)+\operatorname{diag}\left(2 \delta-\varepsilon p_{N} \frac{r_{1} \bar{m}_{1}}{D_{1}}, \ldots, 2 \delta-\varepsilon p_{N} \frac{r_{n} \bar{m}_{n}}{D_{n}}\right) \\
& =\operatorname{diag}\left(\frac{1}{D_{1}}, \ldots, \frac{1}{D_{n}}\right)\left[\operatorname{diag}(\mathbf{v})+\varepsilon p_{N} \iota \tilde{\mathbf{m}}^{\prime}\right]
\end{aligned}
$$

Thus the system of equations (A.12) can be written as $D^{*} \beta=b^{*}$, implying that $\beta=$ $c_{H}\left[\operatorname{diag}(\mathbf{v})+\varepsilon p_{N} \iota \tilde{\mathbf{m}}^{\prime}\right]^{-1} \theta$. Using (A.1) we obtain

$\left[\operatorname{diag}(\mathbf{v})+\varepsilon p_{N} \iota \tilde{\mathbf{m}}^{\prime}\right]^{-1}=\operatorname{diag}(\overline{\mathbf{v}})-\frac{\varepsilon p_{N}}{\mathrm{I}+\varepsilon p_{N} \overline{\mathbf{m}}^{\prime} \overline{\mathbf{v}}} \overline{\mathbf{v}} \tilde{m}^{\prime} \operatorname{diag}(\overline{\mathbf{v}})=\left(\mathbf{I}-\frac{\varepsilon p_{N}}{\chi} \tilde{\mathbf{v}}_{\tilde{\mathbf{m}}} \tilde{\prime}^{\prime}\right) \operatorname{diag}(\overline{\mathbf{v}})$ 
hence

$$
\boldsymbol{\beta}=c_{H}\left(\mathbf{I}-\frac{\varepsilon p_{N}}{\chi} \overline{\mathbf{v}} \tilde{\mathbf{m}}^{\prime}\right) \operatorname{diag}(\overline{\mathbf{v}}) \boldsymbol{\theta}=c_{H}\left(\mathbf{I}-\frac{\varepsilon p_{N}}{\chi} \overline{\mathbf{v}} \overline{\mathbf{m}}^{\prime}\right) \mathbf{w} .
$$

The $\mathrm{i}$-th row of $\mathbf{I}-\left(\varepsilon p_{N} / \chi\right) \overline{\mathbf{v}} \tilde{\mathbf{m}}^{\prime}$ is $\mathbf{e}_{\mathbf{i}}{ }^{\prime}-\left(\varepsilon p_{N} / \chi\right) \bar{v}_{i} \overline{\mathbf{m}}^{\prime}$; thus we obtain

$$
\beta_{i}=c_{H}\left(\frac{\theta_{i}}{v_{i}}-\frac{\varepsilon p_{N}}{v_{i} \chi} \tilde{\mathbf{m}}^{\prime} \mathbf{w}\right)=\frac{c_{H}}{v_{i}}\left(\theta_{i}-\varepsilon p_{N} \frac{\psi}{\chi}\right),
$$

which is just the expression stated in the proposition.

4. We derive $\gamma_{i}$ in several steps. First of all (A.11) implies

$$
2 \delta \gamma_{i}+\varepsilon \sum_{j \neq i} \gamma_{j}=c_{H} \hat{\mathrm{g}}_{\mathrm{i}}-\varepsilon \sum_{j \neq i} \beta_{j} \hat{\mathrm{h}}_{\mathrm{ij}} \quad \forall i
$$

Now define $\Gamma:=\left(\gamma_{1}, \ldots, \gamma_{n}\right)$ and

$$
\mathbf{Z}:=\left(c_{H} \hat{\mathrm{g}}_{\mathfrak{I}}-\varepsilon \sum_{j \neq 1} \beta_{j} \hat{\mathbf{h}}_{\mathbf{1 j}}, \ldots, c_{H} \hat{\mathbf{g}}_{\mathfrak{n}}-\varepsilon \sum_{j \neq n} \beta_{j} \hat{\mathbf{h}}_{\mathfrak{n j}}\right)
$$

Then (using $\tilde{\mathrm{D}}$ as defined in 1.) the system (A.14) can be written as $\Gamma \tilde{\mathbf{D}}=\mathbf{Z}$ or $\Gamma=\mathbf{Z D}^{-1}$. The $\mathrm{i}$-th column of $\overline{\mathbf{D}}^{-1}$ is $(1 / \tilde{d})\left(\mathbf{e}_{\mathbf{i}}-(\varepsilon / \hat{d}) \iota\right)$; therefore

$$
\gamma_{i}=Z \frac{1}{\tilde{d}}\left(\mathbf{e}_{\mathbf{i}}-\frac{\varepsilon}{\hat{d}} \iota\right)=\frac{1}{\tilde{d}}\left[c_{H} \hat{\mathrm{g}}_{\mathrm{i}}-\varepsilon \sum_{j \neq i} \beta_{j} \hat{\mathrm{h}}_{\mathrm{j}}-\frac{\varepsilon}{\hat{d}} \sum_{j=1}^{n}\left(c_{H} \hat{\mathrm{g}}_{\mathrm{j}}-\varepsilon \sum_{k \neq j} \beta_{\mathrm{k}} \hat{\mathrm{h}}_{\mathrm{jk}}\right)\right]
$$

Substituting the expressions for $\hat{\mathrm{g}}_{\mathbf{i}}$ and $\hat{\mathbf{h}}_{\mathrm{ij}}$ given in Proposition 3.2 yields

$$
\begin{aligned}
c_{H} \hat{\mathbf{g}}_{\mathrm{i}}-\varepsilon \sum_{j \neq i} \beta_{j} \hat{\mathrm{h}}_{\mathrm{ij}}= & \frac{1}{D_{i}}\left[c_{H}\left(t_{N} p_{i i}-t_{H} p_{N}\right)-\varepsilon p_{N}\left(p_{i \mathrm{i}}-p_{N}\right) \sum_{j \neq i} \beta_{j} \tilde{m}_{j}\right]\left(\overrightarrow{\mathrm{m}}-\vec{m}_{i} \mathrm{e}_{\mathrm{i}}\right) \\
& -\varepsilon \sum_{j \neq i} \beta_{j}\left(p_{i \mathrm{i}}-p_{N}\right) \tilde{m}_{j} \mathbf{e}_{\mathbf{j}}
\end{aligned}
$$

Since $\sum_{j=1}^{n} \beta_{j} \tilde{m}_{j}=\tilde{\mathbf{m}}^{\prime} \beta=c_{H} \psi\left(1-\varepsilon p_{N} \omega / \chi\right)=c_{H} \psi / \chi$, it follows that

$$
\sum_{j \neq i} \beta_{j} \bar{m}_{j}=\tilde{m}^{\prime} \beta-\tilde{m}_{i} \beta_{i}=c_{H}\left[\frac{\psi}{\chi}-\frac{\tilde{m}_{i}}{v_{i}}\left(\theta_{i}-\varepsilon p_{N} \frac{\psi}{\chi}\right)\right]=\frac{c_{H}}{v_{i}}\left(2 \delta D_{i} \frac{\psi}{\chi}-\tilde{m}_{i} \theta_{i}\right) .
$$

For the [j-term (A.16) we then obtain

$$
\begin{aligned}
c_{H} & \left(t_{N} p_{i i}-t_{H} p_{N}\right)-\varepsilon p_{N}\left(p_{i i}-p_{N}\right) \frac{c_{H}}{v_{i}}\left(2 \delta D_{i} \frac{\psi}{\chi}-\tilde{m}_{i} \theta_{i}\right) \\
& =\frac{c_{H}}{v_{i}}\left[\left(2 \delta D_{i}-\varepsilon p_{N} \tilde{m}_{i}\right)\left(t_{N} p_{i i}-t_{H} p_{N}\right)-\varepsilon p_{N}\left(p_{i i}-p_{N}\right)\left(2 \delta D_{i} \frac{\psi}{\chi}-\tilde{m}_{i} \theta_{i}\right)\right] \\
& =\frac{c_{H} D_{i}}{v_{i}}\left\{2 \delta\left[t_{N} p_{i i}-t_{H} p_{N}-\varepsilon p_{N}\left(p_{i i}-p_{N}\right) \frac{\psi}{\chi}\right]+\varepsilon p_{N} \tilde{m}_{i}\left(t_{H}-t_{N}\right)\right\} \\
& =\frac{c_{H} D_{i}}{v_{i}}\left\{2 \delta\left(p_{i i}-p_{N}\right)\left[t_{H}-\varepsilon p_{N} \frac{\psi}{\chi}+p_{N}\left(t_{H}-t_{N}\right)\left(M-\bar{m}_{i}\right)\right]-\left(t_{H}-t_{N}\right) v_{i}\right\} \\
& =c_{H} D_{i}\left[2 \delta\left(p_{i i}-p_{N}\right) \tilde{\beta}_{i}-\left(t_{H}-t_{N}\right)\right],
\end{aligned}
$$


where $\bar{\beta}_{i}=\beta_{i} / c_{H}$. Substituting this result and

$$
\sum_{j \neq i} \beta_{j}\left(p_{j j}-p_{N}\right) \bar{m}_{j} \mathbf{e}_{\mathbf{j}}=\sum_{j=1}^{n} \beta_{j}\left(p_{j j}-p_{N}\right) \bar{m}_{j} \mathrm{e}_{\mathbf{j}}-\beta_{i}\left(p_{i i}-p_{N}\right) \bar{m}_{i} \mathbf{e}_{\mathbf{i}}
$$

in (A.16) thus yields

$$
\begin{aligned}
& c_{H} \hat{\mathrm{g}}_{\mathrm{i}}-\varepsilon \sum_{j \neq i} \beta_{j} \hat{\mathrm{h}}_{\mathrm{ij}}= {\left[2 \delta\left(p_{i \mathrm{i}}-p_{N}\right) \beta_{\mathrm{i}}-c_{H}\left(t_{H}-t_{N}\right)\right]\left(\overline{\mathrm{m}}-\bar{m}_{\mathrm{i}} \mathbf{e}_{\mathrm{i}}\right) } \\
&-\varepsilon \sum_{j=1}^{n} \beta_{j}\left(p_{j j}-p_{N}\right) \bar{m}_{j} \mathbf{e}_{\mathbf{j}}+\varepsilon \beta_{i}\left(p_{i \mathrm{i}}-p_{N}\right) \bar{m}_{\mathrm{i}} \mathbf{e}_{\mathbf{i}} \quad \text { and } \\
& \sum_{j=1}^{n}\left(c_{H I} \hat{\mathrm{g}}_{\mathrm{j}}-\varepsilon \sum_{k \neq j} \beta_{k} \hat{\mathrm{h}}_{\mathrm{jk}}\right)=\sum_{j=1}^{n}\left[2 \delta\left(p_{j j}-p_{N}\right) \beta_{j}-c_{H}\left(t_{H}-t_{N}\right)\right]\left(\overline{\mathrm{m}}-\bar{m}_{j} \mathbf{e}_{\mathrm{j}}\right) \\
&-(n-1) \varepsilon \sum_{j=1}^{n}\left(p_{j j}-p_{N}\right) \beta_{j} \bar{m}_{j} \mathbf{e}_{\mathrm{j}} .
\end{aligned}
$$

Substituting these expressions in equation (A.15) finally leads to the result given in the proposition.

Proof of Lemma 4.1: The statement of the lemma is equivalent to $t_{H} /\left(v_{i} \chi\right)>0$. Since $\delta>\varepsilon$ and $p_{i i}>p_{N} \forall i, v_{i}$ is positive, hence we only have to show $\chi>0$. According to the definitions of $\chi$ and $\omega$ this is obviously the case for $\varepsilon>0$. Now suppose

$$
\chi=1+\varepsilon p_{N} \sum_{j=1}^{n} \frac{1}{2 \delta p_{i i}-\varepsilon p_{N}}=1+\sum_{j=1}^{n} \frac{1}{2 \delta p_{i i} /\left(\varepsilon p_{N}\right)-1}
$$

were negative for any $\varepsilon<0$. Then the expression would also be negative for $\varepsilon=-\frac{1}{n-1} \delta$, the smallest permissible value. This in turn would imply $\sum_{j=1}^{n} 1 /\left[1+(n-1) p_{j j} / p_{N}\right]>1$. However, this cannot be the case since $p_{j j} / p_{N} \geq 1 \quad \forall j$.

Proof of Proposition 4.1: According to (4.1), $\beta=c_{H} t_{H} /\left(v_{i} \chi\right)$, hence

$$
\frac{\partial \beta_{i}}{\partial p_{i i}}=-\frac{c_{H} t_{H}}{\left(v_{i} \chi\right)^{2}}\left(2 \delta+\varepsilon p_{N} \sum_{j \neq i} \frac{2 \delta}{v_{j}}\right)=-\frac{2 \delta \beta_{i}}{v_{i} \chi}\left(1+\varepsilon p_{N} \sum_{j \neq i} \frac{1}{v_{j}}\right)=-\frac{2 \delta \beta_{i}}{v_{i}}\left(1-\frac{\varepsilon p_{N}}{v_{i} \chi}\right) .
$$

The expression in brackets is always positive for $\varepsilon<0$, since according to Lemma 4.1 $\chi>0$. For positive $\varepsilon$,

$$
\frac{\varepsilon p_{N}}{v_{i} \chi}=\frac{\varepsilon p_{N} / v_{i}}{1+\varepsilon p_{N} \sum_{j=1}^{n}\left(1 / v_{j}\right)} \in(0,1)
$$


Part (a) then follows from Lemma 4.1. Part (b) follows from $E(\pi(s))=\delta p_{i i} \beta_{i}^{2}$, $\partial\left(p_{i \mathrm{i}} \beta_{\mathrm{i}}\right) / \partial p_{i \mathrm{i}} \sim-c_{H}$, and part (a). Part (c) is derived from

$$
\frac{\partial \beta_{i}}{\partial p_{j j}}=\left(-\frac{c_{H} t_{H}}{\left(v_{i} \chi\right)^{2}}\right)\left(-\varepsilon p_{N} \frac{v_{i}}{v_{j}^{2}} 2 \delta\right)=\frac{2 \delta \varepsilon p_{N}}{v_{i} v_{j} \chi} \beta_{j} \sim c_{H} \varepsilon
$$

Finally, noting that $\beta_{i} \beta_{j} \sim c_{H}^{2}>0$ we obtain (d):

$$
\frac{\partial E(\pi(s))}{\partial p_{j j}}=\delta p_{i i} 2 \beta_{i} \frac{\partial \beta_{i}}{\partial p_{j j}}=2 \delta p_{i i} \beta_{i} \frac{2 \delta \varepsilon p_{N}}{v_{i} v_{j} \chi} \beta_{j} \sim \varepsilon
$$

Proof of Lemma 4.2: From $E\left(y_{i}^{2}\right)=p_{H}, E\left[\left(\gamma_{i}^{\prime} \hat{y}\right)^{2}\right]=E\left(\gamma_{i}^{\prime} \hat{y} \hat{y}^{\prime} \gamma_{i}\right)=\gamma_{i}^{\prime} Q \gamma_{i}$ and $E\left(\beta y_{i} \hat{y}^{\prime} \gamma_{i}\right)=\beta \mathbf{p}_{\mathbf{i}}^{\prime} \gamma_{i}$ we obtain

$$
\operatorname{Var}\left(s_{i}\right)=E\left[\left(\beta y_{i}+\boldsymbol{\gamma}_{i}^{\prime} \hat{y}\right)^{2}\right]=p_{H} \beta^{2}+\boldsymbol{\gamma}_{i}^{\prime} \boldsymbol{Q} \boldsymbol{\gamma}_{i}+2 \beta \boldsymbol{p}_{i}^{\prime} \boldsymbol{\gamma}_{i}
$$

Noting that $\mathbf{Q}=\operatorname{diag}(\mathrm{m})+p_{N} \iota \iota^{\prime}$ and $\operatorname{diag}(\mathbf{m})=\left(p_{H}-p_{N}\right) \mathrm{I}$, we compute

$$
\begin{aligned}
\gamma_{i}^{\prime} Q \gamma_{i} & =\left(p_{H}-p_{N}\right) \gamma_{i}^{\prime} \gamma_{i}+p_{N}\left(\iota^{\prime} \gamma_{i}\right)^{2} \\
& =\left(p_{H}-p_{N}\right)\left(\beta-\frac{c_{H}}{\tilde{d}} \theta^{\prime}\right)^{2}\left(\frac{4 n \delta^{2}}{\hat{d}^{2}}-\frac{4 \delta}{\hat{d}}+1\right)+p_{N}\left(\beta-\frac{c_{H}}{\tilde{d}} \theta^{\prime}\right)^{2}\left(\frac{2 n \delta}{\hat{d}}-1\right)^{2} \\
& =\left(\beta-\frac{c_{H}}{\tilde{d}} \theta^{\prime}\right)^{2}\left[\frac{4 \delta}{\hat{d}}\left(\frac{n \delta}{\hat{d}}-1\right) P_{\oplus}+p_{H}\right] \text { and } \\
p_{i}^{\prime} \gamma_{i} & =p_{N} \iota^{\prime} \gamma_{i}+\left(p_{H}-p_{N}\right) e_{i}^{\prime} \gamma_{i} \\
& =p_{N}\left(\beta-\frac{c_{H}}{\tilde{d}} \theta^{\prime}\right)\left(\frac{2 n \delta}{\hat{d}}-1\right)+\left(p_{H}-p_{N}\right)\left(\beta-\frac{c_{H}}{\tilde{d}} \theta^{\prime}\right)\left(\frac{2 \delta}{\hat{d}}-1\right) \\
& =\left(\beta-\frac{c_{H}}{\tilde{d}} \theta^{\prime}\right)\left(\frac{2 \delta}{\hat{d}} P_{\oplus}-p_{H}\right) .
\end{aligned}
$$

with $T_{\oplus}, P_{\oplus}$, and $\theta^{\prime}$ defined as in the lemma. Substituting these expressions in (A.17) yields

$$
\begin{aligned}
\operatorname{Var}\left(s_{i}\right) & =\frac{4 \delta}{\hat{d}} P_{\oplus}\left(\beta-\frac{c_{H}}{\tilde{d}} \theta^{\prime}\right)\left[\frac{n \delta}{\hat{d}}\left(\beta-\frac{c_{H}}{\tilde{d}} \theta^{\prime}\right)+\frac{c_{H}}{\hat{d}} \theta^{\prime}\right]+\frac{b_{H}^{2}}{\tilde{d}^{2}} \theta^{\prime 2} p_{H} \\
& =\frac{4 \delta}{\hat{d}} P_{\oplus}\left(\beta-\frac{c_{H}}{\tilde{d}} \theta^{\prime}\right) \frac{c_{H}}{2 \hat{d} \tilde{d} P_{\oplus}}\left[n \tilde{d} t_{H}+\left(2 \hat{d} P_{\oplus}-n \bar{d}\right) \theta^{\prime}\right]+\frac{b_{H}^{2}}{\tilde{d}^{2}} \theta^{2} p_{H} \\
& =\frac{c_{H}^{2}}{\hat{d}^{2} P_{\oplus}}\left[n t_{H}^{2}-2(n-1) t_{H}\left(t_{H}-t_{N}\right)+\frac{\bar{d}}{\tilde{d}^{2}}\left(n \bar{d}-2 \hat{d} P_{\oplus}\right) \theta^{\prime 2}\right]+\frac{c_{H}^{2}}{\tilde{d}^{2}} \theta^{2} p_{H} \\
& \left.=\frac{c_{H}^{2}}{\hat{d}^{2}}\left[t_{H} \frac{T_{\oplus}}{P_{\oplus}}-(n-1) \frac{t_{H}}{P_{\oplus}}\left(t_{H}-t_{N}\right)+\frac{n \bar{d}^{2}}{\tilde{d}^{2} P_{\oplus}} \theta^{\prime 2}-\frac{2 \hat{d} \bar{d}}{\tilde{d}^{2}} \theta^{\prime 2}+\frac{\hat{d}^{2}}{\tilde{d}^{2}} p_{H} \theta^{\prime 2}\right] A .18\right)
\end{aligned}
$$


The last three terms in (A.18) are equal to

$$
\frac{\theta^{\prime 2}}{\bar{d}^{2}}\left[\frac{n \bar{d}^{2}}{P_{\oplus}}-\hat{d} \bar{d}+(n-1) \hat{d} \varepsilon\left(p_{H}-p_{N}\right)\right]=(n-1) \theta^{2} \frac{p_{H}-p_{N}}{\tilde{d}^{2}}\left[\frac{\tilde{d} \bar{d}}{P_{\oplus}}+\hat{d} \varepsilon\right] \text {. }
$$

After some steps we then obtain

$$
\operatorname{Var}\left(s_{i}\right)=\frac{c_{H}^{2}}{\hat{d}^{2}}\left[t_{H} \frac{T_{\oplus}}{P_{\oplus}}+(n-1) \theta^{\prime}\left(p_{N} \frac{T_{\oplus}}{P_{\oplus}}+\frac{\varepsilon t_{H}-2 \delta t_{N}}{\tilde{d}}+\frac{\hat{d} \varepsilon\left(t_{H}-t_{N}\right)}{\tilde{d}^{2}}\right)\right],
$$

which finally yields the expression given in the Lemma. The covariance is derived analogously, where the expressions obtained are almost identical to those obtained for the variance.

Proof of Proposition 4.3: CV: Since for complete sharing the strategies are identical, $\rho_{s}^{C P}=1$ and $\rho_{s}^{C P}-\rho_{s}^{N P}>0$ follow immediately.

PV: The results follow immediately from $\operatorname{Cov}\left(s_{i}^{C P}, s_{j}^{C P}\right) \sim \varepsilon$ and $\rho_{s}^{N P}=0$.

PS: Again, $\rho_{s}^{C P} \sim \operatorname{Cov}\left(s_{i}, s_{j}\right)$, the sign of which is quickly obtained from Lemma 4.2. Since $\rho_{s}^{N P}=p_{N} / p_{H}$, we have $\rho_{s}^{C P}-\rho_{*}^{N P} \sim p_{H} \operatorname{Cov}\left(s_{i}, s_{j}\right)-p_{N} \operatorname{Var}\left(s_{i}\right)$. This term has the same sign as $\left(p_{H}-p_{N}\right)\left[t_{H}+(n-1) p_{N} \theta^{\prime}\right] \frac{T_{\oplus}}{P_{\oplus}}-(n-1) \theta^{\prime}\left(t_{N} p_{H}-t_{H} p_{N}\right)-\theta\left(t_{H}-t_{N}\right) \frac{4 \delta^{2}+(n-1) \varepsilon^{2}}{\tilde{d}^{2}} P_{\oplus}$, which for perfect signals is $\sim-\varepsilon$.

Proof of Proposition 4.4: 1. CV: Since $t_{H}=t_{N}=t, \theta^{\prime}=0$ and $T_{\oplus}=n t$, the right-hand side of (4.6) reduces to

$$
\delta c_{H}^{2}\left[\frac{1}{\hat{d}^{2}} \cdot\left(t \frac{n t}{P_{\oplus}}\right)-\frac{p_{H} t^{2}}{\tilde{d}^{2}}\right]=\frac{\delta c_{H}^{2} t^{2}}{\hat{d}^{2} \bar{d}^{2} P_{\oplus}}\left(n \bar{d}^{2}-\hat{d}^{2} p_{H} P_{\oplus}\right)
$$

The term in brackets on the right-hand side of (A.19) equals

$$
n\left(\bar{d}^{2}-\hat{d}^{2} p_{H}^{2}\right)+(n-1) \hat{d}^{2} p_{H}\left(p_{H}-p_{N}\right)=(n-1)\left(p_{H}-p_{N}\right)\left(\tilde{d} \hat{d} p_{H}-n \varepsilon \bar{d}\right) .
$$

Decomposing the last term in brackets in (A.20) into the primitive parameters then yields

$$
\begin{aligned}
E\left(\pi_{i}^{C P}\right)-E\left(\pi_{i}^{N P}\right) & =(n-1) \frac{\delta c_{H}^{2} t^{2}}{\hat{d}^{2} \bar{d}^{2} P_{\oplus}}\left(p_{H}-p_{N}\right)\left[4 \delta(\delta-\varepsilon) p_{H}-(n-1) \varepsilon^{2}\left(p_{H}+n p_{N}\right)\right] \\
& \sim 4 \delta(\delta-\varepsilon) p_{H}-(n-1) \varepsilon^{2}\left(p_{H}+n p_{N}\right) \\
& \sim 4 \delta(\delta-\varepsilon)-(n-1) \varepsilon^{2}-n(n-1) \varepsilon^{2} \frac{p_{N}}{p_{H}}
\end{aligned}
$$


Since $p_{N} / p_{H} \in[0,1]$, the last expression is necessarily positive if $4 \delta(\delta-\varepsilon)-(n-1) \varepsilon^{2}>$ $n(n-1) \varepsilon^{2}$ and negative if $4 \delta(\delta-\varepsilon)-(n-1) \varepsilon^{2}<0$. Calculating the critical values of $\varepsilon$ for these inequalities then leads to the result stated in the proposition.

2. $\hat{\mathrm{g}}_{\mathrm{i}}=0$ : From this assumption it follows that $t_{N} p_{H}-t_{H} p_{N}=0$ or $t_{H}\left(p_{H}-p_{N}\right)=$ $p_{H}\left(t_{H}-t_{N}\right)$. Using $t_{H}+(n-1) \theta^{\prime}=P_{\oplus} \theta^{\prime}$, the difference of expected profits given by $(4.4)$ is

$$
\begin{gathered}
\delta c_{H}^{2}\left\{\frac{1}{\tilde{d}^{2}}\left[t_{H}+(n-1)\left(\frac{4 \delta^{2}+(n-1) \varepsilon^{2}}{\tilde{d}^{2}}-1\right)\left(t_{H}-t_{N}\right)\right]-\frac{p_{H} t_{H}^{2}}{\tilde{d}^{2}}\right\} \\
=\frac{\delta c_{H}^{2}}{\tilde{d}^{2} \hat{d}^{2} \bar{d}^{2}} \theta^{\prime}\left[\tilde{d}^{2} \bar{d}^{2} t_{H}+(n-1) \bar{d}^{2} \varepsilon(\tilde{d}+\hat{d})\left(t_{H}-t_{N}\right)-\tilde{d}^{2} \hat{d}^{2} p_{H}^{2} t_{H}\right] \\
=(n-1) \delta \varepsilon \frac{c_{H}^{2}}{\tilde{d}^{2} \hat{d}^{2} \bar{d}^{2}}\left(t_{H}-t_{N}\right) \theta^{\prime}\left[\tilde{d}^{2}(\tilde{d}+\hat{d})-\tilde{d}^{2} t_{H}\left(\vec{d}+\hat{d} t_{H}\right)\right] \\
=(n-1) \delta \varepsilon^{2} \frac{c_{H}^{2}}{\tilde{d}^{2} \hat{d}^{2} \bar{d}^{2}}\left(t_{H}-t_{N}\right) P_{\oplus} \theta^{\prime}\left[\bar{d}(\tilde{d}+\hat{d})+\tilde{d} \hat{d} p_{H}\right]>0 .
\end{gathered}
$$

Proof of Proposition 4.5: 1. We first calculate for a given $\mathrm{k}$ the coefficients $\beta$ and $\gamma_{i}$ for revealing and nonrevealing firms in the general case. Using $R$ as an index for a revealing firm $\mathrm{i}(i=1, \ldots, k)$ and $\mathrm{N}$ as an index for a nonrevealing firm $\mathrm{i}(i=$ $k+1, \ldots, n)$, we obtain $r^{R}=0$ and $r^{N}=\infty ; \bar{m}_{i}^{R}=1 /\left(p_{H}-p_{N}\right)$ and $\bar{m}^{N}=0$, hence $M=k /\left(p_{H}-p_{N}\right)$. In addition,

$$
\begin{aligned}
\theta^{R} & =t_{H}+(k-1) p_{N} \theta^{\prime} & \theta^{N} & =t_{H}+k p_{N} \theta^{\prime} \\
D^{R} & =p_{H}+(k-1) p_{N} & D^{N} & =p_{H}+k p_{N} \\
v^{R} & =2 \delta\left[p_{H}+(k-1) p_{N}\right] & v^{N} & =2 \delta\left(p_{H}+k p_{N}\right)-\varepsilon p_{N} \\
\omega & =\frac{n-k}{2 \delta\left(p_{H}+k p_{N}\right)-\varepsilon p_{N}} & \psi & =\frac{(n-k)\left(t_{H}+k p_{N} \theta^{\prime}\right)}{2 \delta\left(p_{H}+k p_{N}\right)-\varepsilon p_{N}} .
\end{aligned}
$$

Then Proposition 3.3 implies

$$
\begin{aligned}
\beta^{N} & =c_{H} \frac{t_{H}+k p_{N} \theta^{\prime}}{2 \delta\left(p_{H}+k p_{N}\right)+(n-k-1) \varepsilon p_{N}} \\
\beta^{R} & =\frac{c_{H}}{v^{R}}\left(\theta^{R}-\varepsilon p_{N} \frac{\psi}{1+\varepsilon p_{N} \omega}\right)=\frac{c_{H}}{v_{i} \chi}\left[\theta^{R}+\varepsilon p_{N} \frac{n-k}{v^{N}}\left(\theta^{R}-\theta^{N}\right)\right] \\
& =\frac{c_{H}}{2 \delta P_{\oplus}^{k}}\left[\frac{2 \delta\left(p_{H}+k p_{N}\right)-\varepsilon p_{N}}{N^{k}}\left(t_{H}+k p_{N} \theta^{\prime}\right)-p_{N} \theta^{\prime}\right]
\end{aligned}
$$


where $N^{k}:=2 \delta\left(p_{H}+k p_{N}\right)+(n-k-1) \varepsilon p_{N}$ and $P_{\oplus}^{k}:=p_{H}+(k-1) p_{N}$. Furthermore, from Proposition 3.3 we get

$$
\gamma_{i}=\frac{2 \delta}{\tilde{d}}\left(\beta_{i}-\frac{\varepsilon}{\hat{d}} \sum_{j=1}^{n} \beta_{j}-\frac{c_{H}}{\hat{d}} \theta^{\prime}\right) \iota_{k}-\left(\beta_{i}-\frac{c_{H}}{\tilde{d}} \theta^{\prime}\right) \mathrm{e}_{i}^{\mathrm{R}}
$$

where $\iota_{k}$ is a vector with ones in the first $k$ components and zeros in the last $n-k$, and $\mathrm{e}_{\mathrm{i}}^{\mathrm{R}}=\mathrm{e}_{\mathrm{i}}$ if $i \leq k$ and $\mathrm{e}_{i}^{\mathrm{R}}=0$ otherwise.

2. CV: Equation (A.20) and (A.22) imply

$$
\beta^{N}=\frac{c_{H} t_{H}}{N} \quad \text { and } \quad \beta^{R}=\frac{c_{H} t_{H}}{N} \frac{2 \delta\left(p_{H}+k P_{N}\right)-\varepsilon p_{N}}{2 \delta P_{\oplus}^{k}}=\beta^{N}\left(1+\frac{\tilde{d} p_{N}}{2 \delta P_{\oplus}^{k}}\right) .
$$

Then

$\sum_{j=1}^{n} \beta_{j}=\beta^{N}\left(n+\frac{k \tilde{d} p_{N}}{2 \delta P_{\oplus}^{k}}\right) \quad$ and $\beta^{R}-\frac{\varepsilon}{\hat{d}} \sum_{j=1}^{n} \beta_{j}=\frac{1}{\hat{d}} \beta^{N}\left[\tilde{d}+\frac{\tilde{d} p_{N}}{2 \delta \bar{P}_{\oplus}^{k}}(\hat{d}-k \varepsilon)\right]=\frac{\bar{d} N}{2 \delta \hat{d} P_{\Phi}^{k}} \beta^{N}$.

Therefore,

$$
\gamma_{i}^{R}=\frac{2 \delta}{\tilde{d}}\left(\beta^{R}-\frac{\varepsilon}{\hat{d}} \sum_{j=1}^{n} \beta_{j}\right) \iota_{k}-\beta^{R} \mathbf{e}_{\mathbf{i}}=\frac{c_{H} t_{H}}{\hat{d} P_{\oplus}^{k}} \iota_{k}-\beta^{R} \mathbf{e}_{\mathbf{i}} .
$$

Defining $y_{k}$ as the vector of signals $y$ with zeros in the last $(n-k)$ components and noting that $E\left[\left(\iota_{k}^{\prime} y\right)^{2}\right]=\iota_{k}^{\prime} P \iota_{k}=k P_{\oplus}^{k}$, we obtain

$$
s_{i}^{R}=\alpha+\frac{c_{H} t_{H}}{\hat{d} P_{\oplus}^{k}} \iota_{k}^{\prime} y_{k} \quad \text { and } \quad \operatorname{Var}\left(s_{i}^{R}\right)=k \frac{c_{H}^{2} t_{H}^{2}}{\hat{d}^{2} P_{\oplus}^{k}} .
$$

Similar calculations lead to

$$
\gamma_{i}^{N}=\frac{c_{H} t_{H}}{\hat{d} P_{\oplus}^{k}} \frac{2 \delta P_{\oplus}^{k}-k \varepsilon p_{N}}{N} \iota_{k}, \quad s_{i}^{N}=\alpha+\frac{c_{H} t_{H}}{N} y_{i}+\frac{c_{H} t_{H}}{\hat{d} P_{\oplus}^{k}} \frac{2 \delta P_{\oplus}^{k}-k \varepsilon p_{N}}{N} \iota_{k}^{\prime} y_{k} \quad \text { ant }
$$

$\frac{c_{H}^{2} t^{2}}{\left(N^{k}\right)^{2} \hat{d}^{2}} \operatorname{Var}\left(s_{i}^{N}\right)=\left[\hat{d}^{2} p_{H}+k \frac{\left(2 \delta P_{\oplus}^{k}-k \varepsilon p_{N}\right)^{2}}{P_{\oplus}^{k}}+2 k \hat{d} p_{N} \frac{2 \delta P_{\oplus}^{k}-k \varepsilon p_{N}}{P_{\oplus}^{k}}\right]$

noting that $\iota_{k}^{\prime} E\left(\mathrm{y}_{\mathrm{k}} y_{i}\right)=k p_{N}$. Similarly as before, $\Delta \mathrm{E}(\pi):=\mathrm{E}\left(\pi_{i}\left(s_{i}^{R, k+1}, s_{-i}^{\mathbf{k}+\mathbf{1}}\right)\right)-$ $E\left(\pi_{i}\left(s_{i}^{N, k}, s_{-i}^{k}\right)\right)=\delta\left[\operatorname{Var}\left(s_{i}^{R, k+1}\right)-\operatorname{Var}\left(s_{i}^{N, k}\right)\right]$. Hence

$$
\Delta E(\pi) \sim \frac{(k+1) N^{2}}{P_{\oplus}^{k+1}}-\hat{d}^{2} p_{H}-k \frac{\left(2 \delta P_{\oplus}^{k}-k \varepsilon p_{N}\right)^{2}}{P_{\oplus}^{k}}-2 k \hat{d} p_{N} \frac{2 \delta P_{\oplus}^{k}-k \varepsilon p_{N}}{P_{\oplus}^{k}} .
$$

Since $2 \delta P_{\oplus}^{k}-k \varepsilon p_{N}=N-\hat{d} p_{N}$, the first and third terms in (A.24) amount to

$$
k\left(\frac{N^{2}}{P_{\oplus}^{k+1}}-\frac{\left(N-\hat{d} p_{N}\right)^{2}}{P_{\oplus}^{k}}\right)+\frac{N^{2}}{P_{\oplus}^{k+1}}=\frac{k}{P_{\oplus}^{k}} \hat{d} p_{N}\left(2 N-\hat{d} p_{N}\right)+\frac{N^{2}}{P_{\Theta}^{k+1}}\left(1-k \frac{p_{N}}{P_{\Xi}^{k}}\right) .
$$


Substitution into (A.24) yields

$$
\begin{aligned}
\Delta E(\pi) & \sim \frac{\hat{d}^{2}}{P_{\oplus}^{k}}\left(k p_{N}^{2}-P_{\oplus}^{k} p_{H}\right)+\frac{N^{2}\left(p_{H}-p_{N}\right)}{P_{\oplus}^{k} P_{\oplus}^{k+1}} \sim \frac{N^{2}}{P_{\oplus}^{k+1}}-\hat{d}^{2} P_{\oplus}^{k+1} \sim N-\dot{d} P_{\oplus}^{k+1} \\
& =-\varepsilon\left[(n-1)\left(p_{H}-p_{N}\right)+k n p_{N}\right] \sim \sim \varepsilon .
\end{aligned}
$$

3. $\hat{\mathrm{g}}_{\mathrm{i}}=\hat{\mathrm{g}}=0$ : From 1. we obtain

$$
\begin{aligned}
& \beta^{N}=c_{H}\left(t_{H}+k p_{N} \theta^{\prime}\right) / N^{k}=c_{H} P_{\oplus}^{k+1} \theta^{\prime} / N^{k} \text { and } \\
& \beta^{R}=\frac{c_{H}}{2 \delta P_{\oplus}}\left[\frac{2 \delta P_{\oplus}^{k+1}-\varepsilon p_{N}}{N^{k}}\left(t_{H}+k p_{N} \theta^{\prime}\right)-p_{N} \theta^{\prime}\right]=\beta^{N}-c_{H} \frac{\varepsilon}{2 \delta} \frac{p_{N} P_{\oplus}^{n} \theta^{\prime}}{N^{k} P_{\oplus}^{k}}
\end{aligned}
$$

We first calculate $s_{i}^{R}$ and its variance:

$$
\begin{aligned}
\beta^{R} & -\frac{\varepsilon}{\hat{d}} \sum_{j=1}^{n} \beta_{j}-\frac{c_{H}}{\hat{d}} \\
& =\frac{c_{H} \theta^{\prime}}{\hat{d} N^{k}}\left[\tilde{d} P_{\oplus}^{k+1}-N^{k}(2 \delta+(n-k-1) \varepsilon) \frac{\varepsilon}{2 \delta} \frac{p_{N} P_{\oplus}^{n}}{P_{\oplus}^{k}}\right] \\
& =-\frac{c_{H} P_{\oplus}^{n} \varepsilon \theta^{\prime}}{\hat{d} N^{k}}\left[\frac{2 \delta+(n-k-1) \varepsilon}{2 \delta} \frac{p_{N}}{P_{\oplus}^{k}}+1\right]=-c_{H} \frac{\varepsilon}{2 \delta \hat{d}} \frac{P_{\oplus}^{n}}{P_{\oplus}^{k}} \theta^{\prime}
\end{aligned}
$$

Substituting this result in the expression for $\gamma_{i}^{R}$ given by (A.23) leads to $s_{i}^{R}=\alpha+\frac{c_{H}}{\tilde{d}} \theta^{\prime} y_{i}-c_{H} \frac{\varepsilon}{\tilde{d} \hat{d}} \frac{P_{\oplus}^{n}}{P_{\oplus}^{k}} \theta^{\prime} \iota_{k}^{\prime} \mathrm{y}_{\mathrm{k}} \quad$ and $\quad \operatorname{Var}\left(s_{i}^{R}\right)=\frac{c_{H}^{2} \theta^{2}}{\tilde{d}^{2}}\left(p_{H}+k \frac{\varepsilon^{2}}{\hat{d}^{2}} \frac{\left(P_{\oplus}^{n}\right)^{2}}{P_{\oplus}^{k}}-2 \frac{\varepsilon}{\hat{d}} P_{\oplus}^{n}\right)$, noting that $\iota^{\prime} E\left(y_{i} y_{k}\right)=P_{\oplus}^{k}$. The corresponding results for a nonrevealing firm are derived similarly, using $\iota^{\prime} E\left(y_{\mathrm{i}} \mathbf{y}_{\mathbf{k}}\right)=k p_{N}$ :

$$
\begin{aligned}
\gamma_{i}^{N}= & \frac{2 \delta 1}{\tilde{d}}\left(\beta^{N}-\frac{\varepsilon}{\hat{d}} \sum_{j=1}^{n} \beta_{j}-\frac{c_{H} \theta^{\prime}}{\hat{d}}\right) \iota_{k}=-c_{H} \frac{\varepsilon}{\tilde{d} \hat{d}} \frac{P_{\oplus}^{n}}{P_{\oplus}^{k}} \theta^{\prime}\left(1-\frac{\hat{d} p_{N}}{N^{k}}\right) \iota_{k} \\
s_{i}^{N}= & \alpha+c_{H} \frac{P_{\oplus}^{k+1} \theta^{\prime}}{N^{k}} y_{i}-c_{H} \frac{\varepsilon}{\tilde{d} \hat{d}} \frac{P_{\oplus}^{n}}{P_{\oplus}^{k}} \theta^{\prime}\left(1-\frac{\hat{d} p_{N}}{N^{k}}\right) \iota_{k}^{\prime} y_{k} \text { and } \\
\operatorname{Var}\left(s_{i}^{N}\right)= & \frac{c_{H}^{2} \theta^{\prime 2}}{\left(N^{k}\right)^{2} \tilde{d}^{2}}\left[\tilde{d}^{2}\left(P_{\oplus}^{k+1}\right)^{2} p_{H}+k \frac{\varepsilon^{2}}{\hat{d}^{2}} \frac{\left(P_{\oplus}^{n}\right)^{2}}{P_{\oplus}^{k}}\left(N^{k}-\hat{d} p_{N}\right)^{2}\right. \\
& \left.-2 k \varepsilon \frac{\hat{d}}{\hat{d}} \frac{P_{\oplus}^{n}}{P_{\oplus}^{k}} P_{\oplus}^{k+1} p_{N}\left(N^{k}-\hat{d} p_{N}\right)\right]
\end{aligned}
$$

Proceeding similarly as before, we obtain

$$
\Delta E(\pi) \sim\left(N^{k}\right)^{2}\left[p_{H}+(k+1) \frac{\varepsilon^{2}}{\hat{d}^{2}} \frac{\left(P_{\oplus}^{n}\right)^{2}}{P_{\oplus}^{k+1}}-2 \frac{\varepsilon}{\hat{d}} P_{\oplus}^{n}\right]
$$




$$
\begin{aligned}
& -\left[\tilde{d}^{2}\left(P_{\oplus}^{k+1}\right)^{2} p_{H}+k \frac{\varepsilon^{2}}{\hat{d}^{2}} \frac{\left(P_{\oplus}^{n}\right)^{2}}{P_{\oplus}^{k}}\left(N^{k}-\hat{d} p_{N}\right)^{2}-2 k \varepsilon \frac{\tilde{d}}{\dot{d}} P_{\oplus}^{k+1} \frac{P_{\oplus}^{n}}{P_{\oplus}^{k}} p_{N}\left(N^{k}-\hat{d} p_{N}\right)\right] \\
= & {\left[\left(N^{k}\right)^{2}-\tilde{d}^{2}\left(P_{\oplus}^{k+1}\right)^{2}\right] p_{H}+\frac{\varepsilon^{2}}{\dot{d}^{2}}\left(P_{\oplus}^{n}\right)^{2}\left[(k+1) \frac{\left(N^{k}\right)^{2}}{P_{\oplus}^{k+1}}-k \frac{\left(N^{k}-\hat{d} p_{N}\right)^{2}}{P_{\oplus}^{k}}\right] } \\
& -2 \frac{\varepsilon}{\hat{d}} P_{\oplus}^{n}\left[\left(N^{k}\right)^{2}-k \tilde{d} P_{\oplus}^{k+1}\left(N^{k}-\hat{d} p_{N}\right) \frac{p_{N}}{P_{\oplus}^{k}}\right]
\end{aligned}
$$

The terms in square brackets in (A.25) are

$$
\begin{gathered}
\left(N^{k}+\tilde{d} P_{\oplus}^{k+1}\right) \varepsilon P_{\oplus}^{n}, \quad \frac{k \hat{d} p_{N}\left(2 N^{k}-\hat{d} p_{N}\right)}{P_{\oplus}^{k}}+\frac{\left(N^{k}\right)^{2}\left(p_{H}-p_{N}\right)}{P_{\oplus}^{k} P_{\oplus}^{k+1}} \text { and } \\
\frac{1}{P_{\oplus}^{k}}\left(\left(N^{k}\right)^{2}\left(p_{H}-p_{N}\right)+k \varepsilon N^{k} p_{N} P_{\oplus}^{n}+k \tilde{d} \hat{d} P_{\oplus}^{k+1} p_{N}^{2}\right)
\end{gathered}
$$

respectively. Substitution into (A.25) yields

$$
\begin{aligned}
\frac{\Delta E(\pi)}{\varepsilon} \sim & \left(N^{k}+\tilde{d} P_{\oplus}^{k+1}\right) p_{H}-k \varepsilon p_{N}^{2} \frac{P_{\oplus}^{n}}{P_{\oplus}^{k}}+\frac{\varepsilon}{\hat{d}^{2}} \frac{P_{\oplus}^{n}}{P_{\oplus}^{k} P_{\oplus}^{k+1}}\left(N^{k}\right)^{2}\left(p_{H}-p_{N}\right) \\
& -2 \frac{\left(N^{k}\right)^{2}}{\hat{d} P_{\oplus}^{k}}\left(p_{H}-p_{N}\right)-2 k \tilde{d} \frac{P_{\oplus}^{k+1}}{P_{\oplus}^{k}} p_{N}^{2} \\
\sim & \left(p_{H}-p_{N}\right)\left[\left(2 \tilde{d} P_{\oplus}^{k+1}+\varepsilon P_{\oplus}^{n}\right) \frac{P_{\oplus}^{k+1}}{P_{\oplus}^{k}}+\frac{\left(N^{k}\right)^{2}}{\hat{d} P_{\oplus}^{k}}\left(\frac{\varepsilon P_{\oplus}^{n}}{\hat{d} P_{\oplus}^{k+1}}-2\right)\right] \\
\sim & \left(2 \tilde{d} P_{\oplus}^{k+1}+\varepsilon P_{\oplus}^{n}\right) P_{\oplus}^{k+1}+\frac{\left(N^{k}\right)^{2}}{\hat{d}^{2} P_{\oplus}^{k+1}}\left(\varepsilon P_{\oplus}^{n}-2 \hat{d} P_{\oplus}^{k+1}\right) \\
\sim & 2 \tilde{d} \hat{d}^{2}\left(P_{\oplus}^{k+1}\right)^{3}+\hat{d}^{2} \varepsilon P_{\oplus}^{n}\left(P_{\oplus}^{k+1}\right)^{2}+\left(N^{k}\right)^{2} \varepsilon P_{\oplus}^{n}-2 \hat{d}\left(N^{k}\right)^{2} P_{\oplus}^{k+1} \\
= & 2 n \tilde{d} \hat{d} \varepsilon\left(P_{\oplus}^{k+1}\right)^{3}+\hat{d}^{2} \varepsilon P_{\oplus}^{n}\left(P_{\oplus}^{k+1}\right)^{2}+\left(N^{k}\right)^{2} \varepsilon P_{\oplus}^{n}-2 \hat{d} \varepsilon N^{k} P_{\oplus}^{k+1} P_{\oplus}^{n}-2 \tilde{d} \hat{d} \varepsilon\left(P_{\xi}^{k+1}\right)^{2} P_{\sqsubseteq}^{n},
\end{aligned}
$$

hence

$$
\Delta E(\pi) \sim 2 \tilde{d} \hat{d}\left(P_{\oplus}^{k+1}\right)^{2}\left[(n-1)\left(p_{H}-p_{N}\right)+k n p_{N}\right]+P_{\oplus}^{n}\left(\hat{d} P_{\oplus}^{k+1}-N^{k}\right)^{2}
$$

where both terms are positive. 


\section{B Definitions of symbols}

symbol definition/meaning

$a_{i}($.$) \quad term in profit function$

$b_{i i} \quad$ parameter of profit function

$b_{N} \quad$ parameter of profit function

$b_{H} \quad b_{i i}$ in the symmetric model

$c_{H} \quad$ parameter of profit function

$c_{N} \quad$ parameter of profit function

$\tilde{d} 2 \delta-\varepsilon$

$\hat{d} \quad 2 \delta+(n-1) \varepsilon$

$\bar{d} \quad 2 \delta p_{H}+(n-1) \varepsilon p_{N}$

$e_{i} \quad \mathrm{i}$-th unit vector

$g_{i} \quad$ coefficient of $y_{i}$ in $E\left(\tau_{i} \mid z_{i}\right)$

$\hat{\mathrm{g}}_{\mathrm{i}} \quad$ coefficient vector of $\hat{\mathrm{y}}$ in $E\left(\tau_{i} \mid \mathbf{z}_{\mathbf{i}}\right)$

$h_{i j} \quad$ coefficient of $y_{i}$ in $E\left(y_{j} \mid \mathbf{z}_{\mathbf{i}}\right)$

$\hat{\mathrm{h}}_{\mathrm{ij}} \quad$ coefficient vector of $\hat{\mathrm{y}}$ in $E\left(y_{j} \mid \mathbf{z}_{\mathbf{i}}\right)$

$m_{i} \quad p_{i i}-p_{N}+r_{i}$

$\bar{m}_{i} \quad 1 / m_{i}$

$m \quad m_{i}$ in the symmetric model

$\tilde{m}_{i} \quad r_{i} \bar{m}_{i}$

$n$ number of firms

$p_{i i} \quad t_{H}+u_{i i}$, variance of $y_{i}$

$p_{N} \quad t_{N}+u_{N}$, covariance of the $y_{i}$

$p_{H} \quad p_{i i}$ in the symmetric model

$p_{i} \quad i$-th column vector of $\mathbf{P}$

$r_{i} \quad$ variance of $\xi_{i}$, revelation strategy

$s_{i} \quad$ strategy of firm $i$ in oligopoly game

$t_{H} \quad$ variance of $\tau_{i}$

$t_{N} \quad$ covariance of the $\tau_{i}$

$t_{i} \quad i$-th column vector of $T$

$u_{i i} \quad$ variance of signal error $\eta_{i}$

$u_{N} \quad$ covariance of the signal errors $\eta_{i}$

$u_{H} \quad u_{i i}$ in the symmetric model

$\bar{v}_{i} \quad 1 / v_{i}$ 
symbol definition/meaning

$v_{i} \quad 2 \delta D_{i}-\varepsilon p_{N} r_{i} \vec{m}_{i}$

$w_{i} \quad \theta_{i} / v_{i}$

$y_{i} \quad \tau_{i}+\eta_{i}$, signal firm i receives

$\hat{y}_{i} \quad y_{i}+\xi_{i}$, i's revealed signal

$z_{i} \quad\left(y_{i}, \hat{y}\right)^{\prime}$, vector of information variables

D $\quad \delta \mathrm{I}+\varepsilon \overline{\mathbf{I}}$, parameter matrix of profit function

$D_{i} \quad p_{i i}+p_{N}\left(p_{i i}-p_{N}\right)\left(M-\bar{m}_{i}\right)$

I n-dimensional unit matrix

$M \quad \iota^{\prime} \overline{\mathrm{m}}$

$N^{k} \quad 2 \delta\left(p_{H}+k p_{N}\right)+(n-k-1) \varepsilon p_{N}$

$\mathbf{P} \quad \mathbf{T}+\mathbf{U}$, covariance matrix of $\mathbf{y}$

$P_{\oplus} \quad p_{H}+(n-1) p_{N}$

$P_{\oplus}^{k} \quad p_{H}+(k-1) p_{N}$

$\mathbf{Q} \quad \mathbf{T}+\mathbf{U}+\operatorname{diag}(\mathbf{r})=\mathbf{P}+\operatorname{diag}(\mathbf{r})$, covariance matrix of $\hat{\mathbf{y}}$

$T \quad$ covariance matrix of $\tau$

$T_{\oplus} \quad t_{H}+(n-1) t_{N}$

$T_{\oplus}^{k} \quad t_{H}+(k-1) t_{N}$

$\mathrm{U}$ covariance matrix of $\boldsymbol{\eta}$

$\alpha_{i} \quad$ coefficient of equilibrium strategy $s_{i}$

$\beta_{i} \quad$ coefficient of $y_{i}$ in equilibrium strategy $s_{i}$

$\beta \quad \beta_{i}$ in the symmetric model

$\gamma_{i} \quad$ coefficient vector of $\hat{y}$ in equlibrium strategy $s_{i}$

$\delta \quad$ parameter of profit function

$\varepsilon \quad$ parameter of profit function

$\eta_{i} \quad$ error term in i's private signal

$\theta_{i} \quad t_{H}+p_{N}\left(p_{i i}-p_{N}\right)\left(M-\bar{m}_{i}\right)$

$\theta^{\prime} \quad\left(t_{H}-t_{N}\right) /\left(p_{H}-p_{N}\right)$ (in the symmetric model)

$\iota \quad(1, \ldots, 1)^{\prime}$

$\xi_{i} \quad$ error term in revealed signal $\hat{y}_{i}$

$\pi_{i} \quad$ profit of firm i

$\rho_{a} \quad$ correlation coefficient of components of a (for any vector a)

$\tau_{i} \quad$ i-th component of State of Nature

$\chi \quad 1+\varepsilon p_{N} \omega$

$\psi \quad \tilde{m}^{\prime} w$

$\omega \quad \overline{\mathrm{m}}^{\prime} \overline{\mathrm{v}}$

$\Gamma \quad\left(\gamma_{1}, \ldots, \gamma_{n}\right)$ 


\section{References}

Basar, Tamer and Yu-Chi Ho 1974: Informational Properties of the Nash Solutions of

Two Stochastic Nonzero-sum Games; Journal of Economic Theory 7: 370-387

Bulow, J.; John Geanakoplos and Paul Klemperer 1985: Multimarket Oligopoly: Strategic

Substitutes and Complements; Journal of Political Economy 93: 488-511

Clarke, Richard 1983: Collusion and the Incentives for Information Sharing; Bell Journal of

Economics 14: $383-394$

Fried, Dov 1984: Incentives for Information Production and Disclosure in a Duopolistic

Environment; Quarterly Journal of Economics 99: 367-381

Gal-Or, Esther 1985: Information Sharing in Oligopoly; Econometrica 53: 329-343

1986: Information Transmission - Cournot and Bertrand Equilibria; Review of Econo-

mic Studies 53: 85-92

Hviid, Morten 1989: Risk-averse Duopolists and Voluntary Information Transmission; Journal of Industrial Economics 38: 49-64

Kirby, Alison J. 1988: Trade Associations as Information Exchange Mechanisms; RAND Journal of Economics 19: 138-146

$\mathrm{Li}$, Lode 1985: Cournot Oligopoly with Information Sharing, RAND Journal of Economics 16: $521-536$

Li, Lode; Richard D. McKelvey and Talbot Page 1987: Optimal Research for Cournot Oligopolists; Journal of Economic Theory 42: 140-166

Madansky, Albert 1976: Foundations of Econometrics; Amsterdam and Oxford

Monderer, Dov and Lloyd Shapley 1991: Potential Games, mimeo

Novshek, William and Hugo Sonnenschein 1982: Fulfilled Expectations Cournot Duopoly

with Information Acquisition and Release, Bell Journal of Economics 13: 214-218

Okuno-Fujiwara, Masahiro; Andrew Postlewaite and Kotaro Suzumura i990: Strategic In-

formation Revelation; Review of Economic Studies 57: 25-47

Ponssard, Jean-Pierre 1979: The Strategic Role of Information on the Demand Function in an Oligopolistic Market; Management Science 25: 243-250

Radner, Roy 1962: Team Decision Problems; Annals of Mathematical Statistics 33: 857-81 
Sakai, Yasuhiro 1986: Cournot and Bertrand Equilibria Under Imperfect Information; Journal of Economics (Zeitschrift für Nationalökonomie) 46: 213-32

Sakai, Yasuhiro and Takehiko Yamato 1989: Oligopoly, Information and Welfare; Journal of Economics (Zeitschrift für Nationalokonomie) 49: 3-24

Schōnfeld, Peter 1969: Methoden der Ökonometrie Bd. I; Berlin and Frankfurt a.M.

Shapiro, Carl 1986: Exchange of Cost Information in Oligopoly; Review of Economic Studies 53: $433-446$

Theil, Henri 1971: Principles of Econometrics; Santa Barbara et.al.

Vives, Xavier 1984: Duopoly Information Equilibrium: Cournot and Bertrand; Journal of Economic Theory 34: 71-94

_- 1988: Aggregation of Information in Large Cournot Markets; Econometrica 56: 851876

1990: Trade Association Disclosure Rules, Incentives to Share Information, and Welfare; RAND Journal of Economics 21: 409-430 